\title{
A mission concept for the low-cost large-scale exploration and characterisation of near earth objects
}

\author{
Lewis Walker ${ }^{\mathrm{a}, *}$, Marilena Di Carlo ${ }^{\mathrm{a}}$, Cristian Greco ${ }^{\mathrm{a}}$, Massimiliano Vasile ${ }^{\mathrm{a}}$, \\ Matthew Warden ${ }^{\mathrm{b}}$ \\ ${ }^{a}$ Level 8, James Weir Building, 75 Montrose St, Glasgow G1 1XJ, UK \\ ${ }^{\mathrm{b}}$ Level 5, Technology and Innovation Centre, 99 George Street, Glasgow G1 IRD, UK
}

Received 9 April 2020; received in revised form 21 October 2020; accepted 23 October 2020

\begin{abstract}
This paper presents the preliminary mission and science analysis of a new mission concept for the large scale, low-cost exploration of Near Earth Objects (NEOs). The concept is to enable close range observations of NEOs by performing close flybys of a series of NEOs at one of their nodal points, with pairs of small spacecraft flying in formation. The paper presents a preliminary assessment of accessible asteroids and multi-target tour trajectories from data available in the JPL small-body database.

The main instruments on board each spacecraft are a camera and a LIDAR which together can be used for orbit determination, surface imaging, direct asteroid ranging and asteroid mass estimation via intersatellite ranging. The paper provides a qualitative and quantitative assessment of the measurable quantities during each flyby. In particular, the feasibility of a novel method of NEO mass estimation is assessed.
\end{abstract}

(C) 2020 Published by Elsevier Ltd on behalf of COSPAR.

Keywords: Near Earth asteroid; Formation flight; Flyby; Radioscience

\section{Introduction}

Near-Earth Objects (NEOs) are defined as objects whose perihelion is less than 1.3 astronomical units (au). The majority of NEOs are asteroids (NEAs). As of April 2nd 2020, 22,534 NEOs have been identified by astronomers and space agencies worldwide (Center for Near Earth Object Studies, 2019). Observations from Earth and satellites in Earth orbit have progressively increased our knowledge of the NEO population and more are constantly being discovered. However, only 20 NEOs have been visited by spacecraft to date (JPL Small Body Database, 2019), with many of these being distant flybys, thus the NEO population remains rela-

\footnotetext{
* Corresponding author.

E-mail address: lewis.walker@strath.ac.uk (L. Walker).
}

tively unexplored. Table 1 shows the cost associated with various past and current space missions to asteroids and comets, not adjusted for inflation (NASA SSDC Archive, 2020).

In a previous work by the authors (Greco et al., 2018), it was shown that for rendezvous missions, the propellant and time cost limits the number of reachable NEOs to those with low eccentricity and low inclination. High inclination and eccentricity NEOs (deep crossers) are hence the natural target of a deflection action with a kinetic impactor, as explained in Thiry and Vasile (2017). Therefore from a planetary defence perspective, deep crossers are interesting to explore in order to have better confidence in the effectiveness of a kinetic impactor. Obviously for any redirection attempt, the asteroid mass is an important parameter to know, and this mission concept makes mass estimation a primary objective. 
Table 1

Examples of past and current missions to asteroids and comets.

\begin{tabular}{ccc}
\hline Mission & Total Cost $(\mathrm{M} \$)$ & \# visited objects \\
\hline NEAR Shoemaker & 220.5 & 2 \\
Stardust & 199.6 & 3 \\
Deep Space 1 & 152.3 & 2 \\
Deep Impact & 280 & 2 \\
Hayabusa & 100 & 1 \\
Hayabusa2 & 150 & 1 \\
Rosetta & 975 & 3 \\
Dawn & 446 & 2 \\
\hline
\end{tabular}

From Greco et al. (2018) it was clear that if any significant portion of the NEO population was to be explored, current mission concepts relying on a single orbiter visiting a single target should be replaced with lower cost options that allow the exploration of many objects with a single mission. The need to reduce the cost would suggest the use of multi-purpose standard platforms that could be built in series and reconfigured to adapt to a range of targets. Cost of launch and operations also need to be reduced to allow frequent visiting and revisiting of different NEOs.

The question of planetary defence is not the only one driving the exploration of NEOs however. Science and asteroid exploitation are other two aspects that motivate a mission to NEOs, where science is certainly what has made the most compelling case so far. Thus there is generally the need to maximise the scientific return of a mission. Examples of current mission proposals, however, have been built around a compromise between cost, scientific return, planetary defence needs and completion time (Snodgrass and Jones, 2019; Machuca et al., 2020).

In the same spirit, and following the results in Greco et al. (2018), this paper presents a mission concept to visit several NEOs that represents a compromise between low cost exploration and overall scientific return. The goal is to extract as much information as possible from fast flybys of multiple objects to maximise scientific return. In order to fulfil the primary objective of mass estimation, the method we propose requires the use of twin spacecraft flying by each asteroid simultaneously. The flight profile, already formulated in Carlo et al. (2017) and Greco et al. (2018), is to flyby multiple NEOs at their nodal points with the ecliptic. This choice avoids expensive changes of inclination and makes the propellant and time cost only dependent on the distance of the nodal point from the Sun and the time at which the NEO is flying through the nodal point.

The main question is what information about each target can be extracted during a fast flyby - in particular which critical pieces of information can be gained from a close approach that cannot be recovered from Earth. As shown in Vetrisano and Vasile (2016) one can improve the ephemerides of asteroids during close approach. The orbital parameters of many NEOs are only known with limited accuracy, hence pin-pointing the exact flyby time and location is not possible in advance without additional observa- tions, making orbit determination (OD) a requirement for any proximity measurements. For close range measurements, the mass and shape of the NEO are extremely important to make any prediction on the evolution of the orbit and the effectiveness of a deflection action. While the shape can be partially reconstructed from appropriate observations from Earth, accurate estimation of the mass would require an in-situ experiment. Furthermore, a reconstruction of the shape is not easily achievable for all NEOs from Earth. Additionally to this, surface imaging would be useful scientific data as this will give insight into the composition and structure. This is also relevant to potential kinetic impactors as the structure of the object can affect the response to an impactor.

Thus this paper proposes a strategy to improve the knowledge of the orbit, estimate of the mass, and recover images to determine the three-dimensional shape and structure of the target objects.

The main contribution of this paper is the proposed mission concept to acquire useful scientific and planetary defence information on a large number of NEOs using multiple flybys. This goal can be achieved through the multi-asteroid tour trajectory and fast-flyby metrology approach proposed in this paper.

Some existing missions and concepts similar to the concept that will be presented in this paper include NASA's planned NEA Scout (McNutt et al., 2014), a low-cost CubeSat close-flyby mission to an as of yet undetermined asteroid target, and JAXA's PROCYON (Funase et al., 2014), another CubeSat asteroid flyby mission, which was launched in 2014 but later abandoned due to a malfunction. However, no similar missions to the author's knowledge make attempts to determine asteroid mass, which is one of the key scientific returns of our concept.

The first part of the paper introduces the overall mission concept, the selection of possible asteroid sequences and the design of the associated trajectory. This was the result of a Concurrent Engineering study, in collaboration with the European Space Agency (ESA), the Observatoire de Paris and the Centre National d'études Spatiales (CNES), and follows a number of requirements provided by ESA. It was decided that the two spacecraft would be of the same class as the M-ARGO mission (Walker et al., 2017), with a similar type of propulsion system and a maximum lifetime of 3 years. This analysis is essential to derive realistic numbers on the speed of flyby and on the targets that can be visited in a given time frame. The mission concept is called NEACORE - Nanospacecraft Exploration of Asteroids by COllision and flyby REconnaissance.

The second part of the paper starts from the navigation requirements during close approach, then focuses on the payloads, with modelling of the camera and LIDAR instrument and the overall mass estimation strategy during flyby, in Section 5. Results show the accuracy of the mass estimation depends strongly on the flyby distance, asteroid mass and flyby velocity. Finally, Section 7 concludes the paper with some final remarks. 


\section{Mission concept}

The mission concept is to have multiple pairs of spacecraft with each pair embarking on a unique asteroid flyby tour. The primary mission objective is to obtain better measurements of asteroids than can be obtained from Earth-based observations, of primarily mass and orbital elements, but also structure, size, and shape. We considered spacecraft with a maximum mass of $24 \mathrm{~kg}$ and the stowed volume and shape of a $12 \mathrm{U}$ CubeSat. The overall conceptual system design (Walker et al., 2019) took inspiration from the ESA M-ARGO mission but with a different payload. Each spacecraft is equipped with an ion engine that is used for the realisation of the tour.

For each pair it is possible to identify a different sequence of target asteroids as shown in Greco et al. (2018). In order to maximise the number of asteroids and allow continuous exploration of NEOs we considered different launch scenarios: a dedicated launch of minimum two spacecraft with an escape $\mathrm{C} 3=0$ (i.e. the asymptotic velocity of the escape trajectory is zero), a dedicated launch of multiple pairs in a single stack on an escape trajectory with $\mathrm{C} 3=0$ and a piggy-back launch of a pair or stack to the Earth-Sun L2 point.

The first option considered the use of current and future generations of nanolaunchers. Out of an initial list including more than 100 existing and planned launchers we down-selected LauncherOne (Virgin Orbit Service Guide, 2019) and Skyrora XL (information obtained via direct email communication with the company), which were the two cheapest options able to launch a single pair with a reasonable mass margin. In this case the upper stage must be re-designed or designed to allow an injection onto an escape trajectory. The mass margin was accounted after a preliminary design of the upper stage, thus the selected vehicles can be considered a robust choice.

For the second option we considered larger launchers which could put a stack of spacecraft in a heliocentric trailing orbit from which each pair could be deployed and begin their tour. The vehicles selected were the PSLV-CA (PSLVSpecifications, 2019), capable of launching 3 pairs plus a dedicated upper stage at a launch cost of $31 \mathrm{M} \$$, the Epsilon (Epsilon Launch Vehicle User's Manual, 2019), capable of launching 6 pairs plus upper stage at a launch cost of $39 \mathrm{M} \$$, and for the most extreme case the Falcon 9 FT expendable configuration (Falcon User's Guide, 2019), capable of launching 95 pairs plus an upper stage at a launch cost of $62 \mathrm{M} \$$. In this case the idea is to station a stack of spacecraft at L2 and release each pair when a transfer window to its tour is available.

In Walker et al. (2019) a cost analysis was performed for the NEACORE concept assuming a dedicated launch of 3 pairs of satellites with a kick stage aboard the PSLV-CA. The total cost of such a launch including 4 years and 8 months of ground support was estimated to be as low as 34 million USD, or 5.7 million USD per spacecraft.

An illustration of the overall mission can be seen in Fig. 1, and a detailed Concept of Operations for the flyby

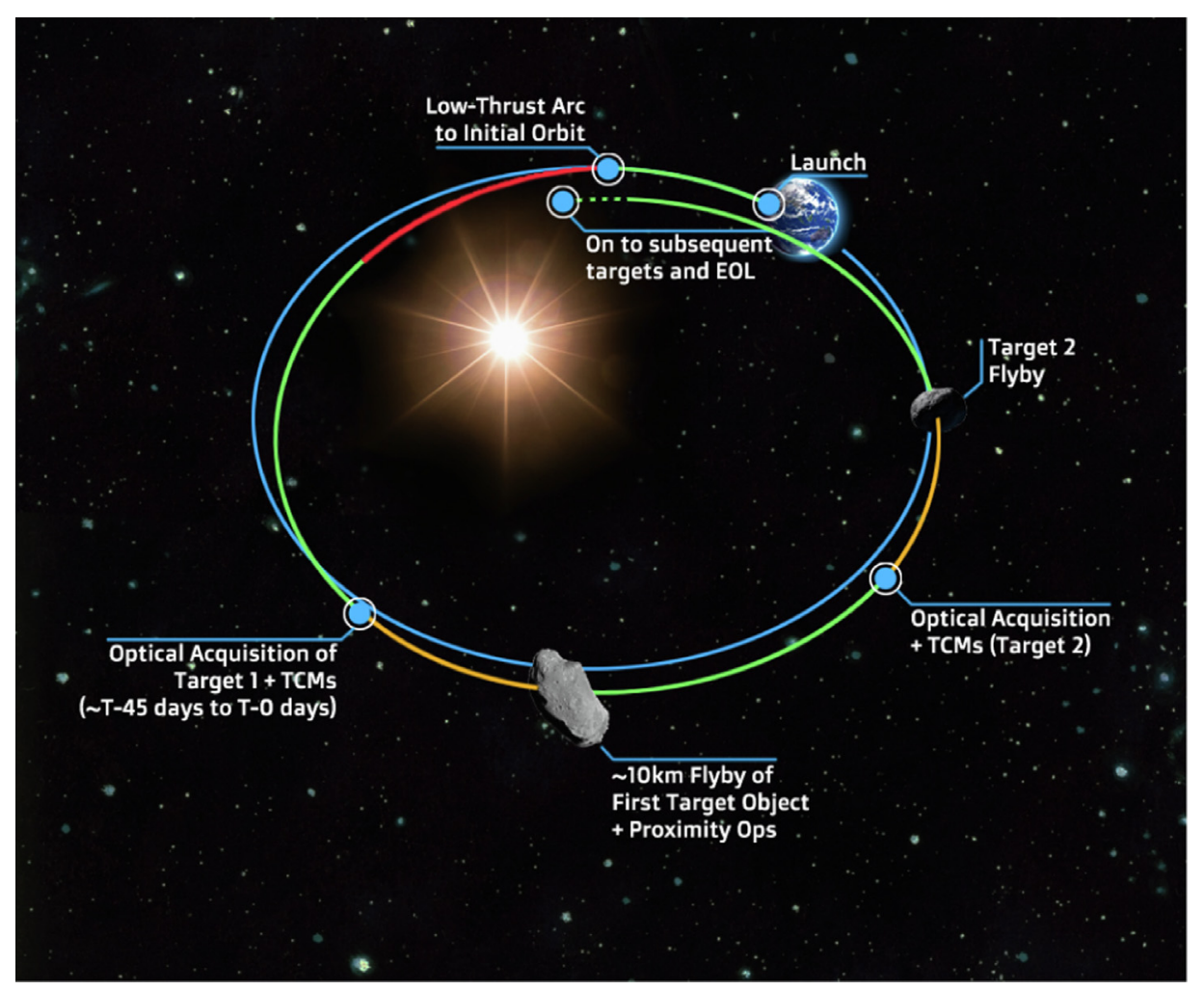

Fig. 1. Illustration of the low-thrust multi-target close-flyby tour concept. 


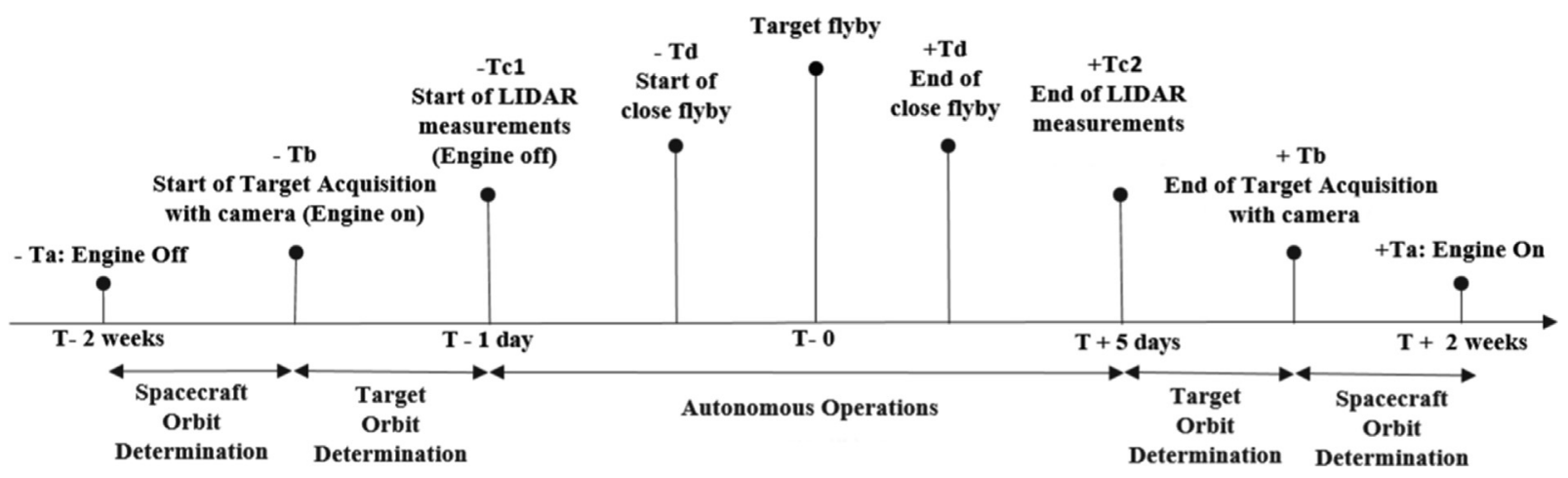

Fig. 2. CONOPS for the Observation phase.

phase can be found in Fig. 2. On approach to each target, the two spacecraft use their cameras to acquire images of the target and improve the knowledge of its orbit. This information is used to re-target the point of close approach and to calculate a number of trajectory correction manoeuvres (TCMs) in the final weeks of approach that allow the two spacecraft to achieve a very close flyby. It should be noted that given the imprecisely known orbit and mass of the NEO the targeted flyby distance is only an estimation, and as will be explained later in the paper the actual flyby distance will depend on the uncertainty on the orbital elements and mass of the NEO at the end of the OD/TCM phase.

Before, during and after the flyby, the cameras are used to determine the shape and angular size of the object, as well as obtain images of the surface. As the spacecraft separation will be known, cameras from two perspectives on approach will give the distance to the object and allow calculation of physical size. On-board LIDAR (light detection and ranging) instruments, one per spacecraft, are used to precisely monitor the separation of the two spacecraft during and after the flyby.

During the close approach, the asteroid's gravity will deflect the trajectories of the two spacecraft slightly compared with their unperturbed trajectories. By measuring their relative position after the flyby with the LIDARs and comparing with the unperturbed prediction, the mass of the object can be estimated.

After the end of the mass estimation phase, the two spacecraft enter a second OD phase until either the asteroid is too distant for this to continue, or the next thrust arc commences. After flyby, engines remain off until the next burn which alters the trajectory for a flyby with the next asteroid in the tour.

Since all mission scenarios would practically correspond to a start of the asteroid tour with a $\mathrm{C} 3=0$, in the following we will present an example of trajectory design that is applicable to all three mission scenarios.

\section{Trajectory design and target selection}

In Greco et al. (2018) it was shown that multiple lowthrust trajectory options are available to visit individual
NEOs, for either rendezvous or flyby. In this paper it will be shown that there exist many trajectories that allow the flyby of multiple NEOs in a single tour. If one imposes no limitations on the size of the NEO and accepts a mission lifetime of over seven years, there can be up to 15 NEOs in a single tour. However, when constraints on the total transfer time and the minimum size of the asteroids are introduced, the number of asteroids in the set of possible tours is reduced to less than five per tour. Details of the different possible solutions for different years, and a nominal reference solution, are presented in this section.

In the remainder, it is assumed that the spacecraft remains in the ecliptic plane and performs flybys of the asteroids at their nodal points, thus avoiding expensive out-of-plane maneuvers and reducing the total propellant requirement. The transfer and correction maneuvers required to allow the flybys are performed using a lowthrust electric propulsion engine, whose maximum thrust is assumed to be inversely proportional to the square of the distance from the Sun. The thrust level at 1 Astronomical Unit (au) is assumed to be $3 \mathrm{mN}$, with a specific impulse $I_{s p}=3000 \mathrm{~s}$. These performance numbers were taken from the Ariane Group's RIT $\mu \mathrm{X}$ ion thruster (Leiter et al., 2015). Fig. 3 shows, schematically, the process followed to obtain the final low-thrust solution. The different steps of the process will be presented in more details in the next subsections.

\subsection{Flyby sequence selection}

In order to determine a sequence of asteroids that can be visited in a given time frame, first we considered only the subpopulation of NEOs with an estimated diameter lower than $500 \mathrm{~m}$. The reason for this choice is that the majority of large asteroids can be well observed from Earth and there are far fewer of these than smaller asteroids. Asteroids with a diameter of $500 \mathrm{~m}$ or lower still represent a non-negligible threat for life on Earth. Furthermore, the smaller the asteroid, the less information we have and can be acquired with observations from Earth, increasing the need for proximity observations. Fig. 4 shows the distribution of orbital elements of NEOs in the entire JPL small body database (JPL Small Body Database, 2019) as 


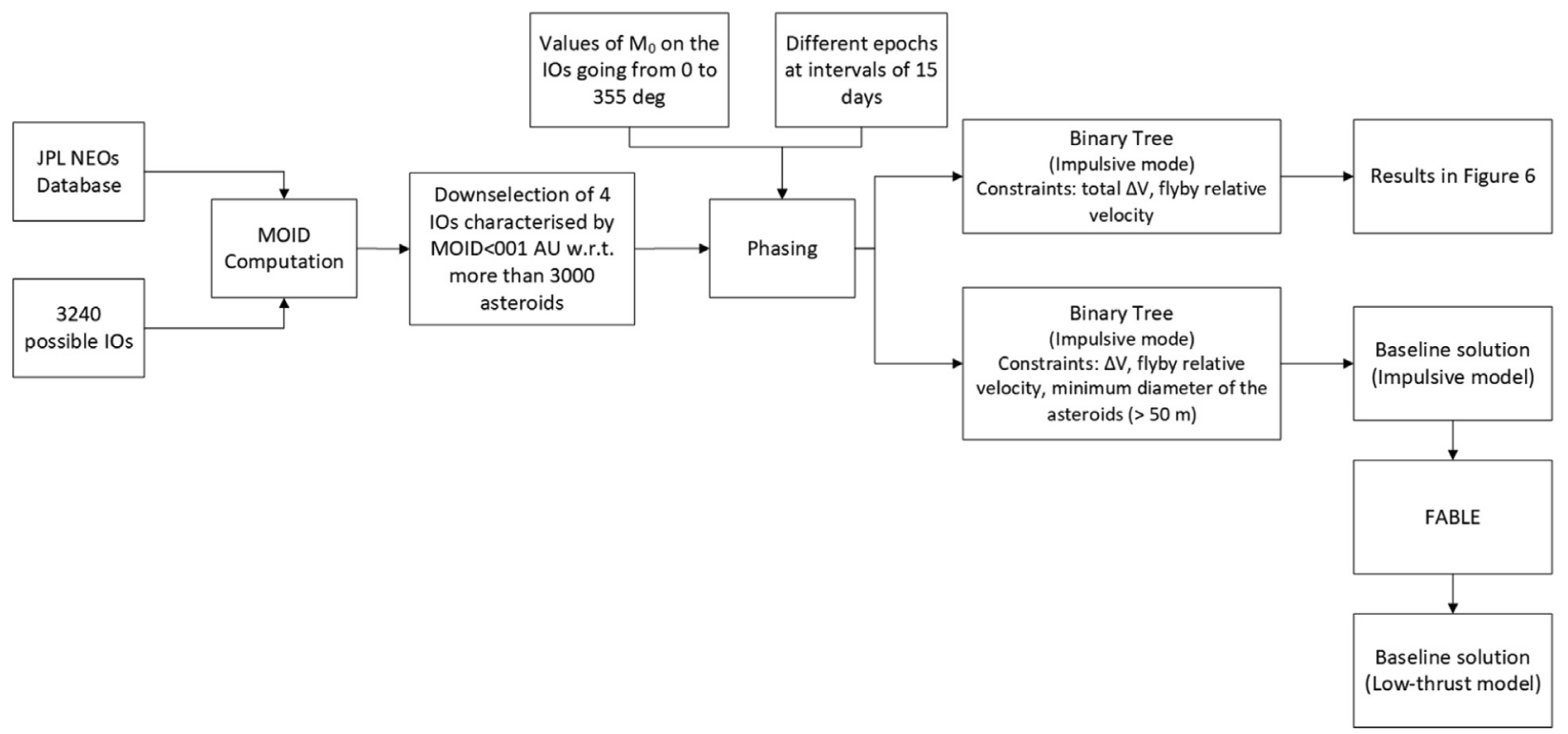

Fig. 3. Schematic representation of the process followed to obtain the low-thrust reference tour.
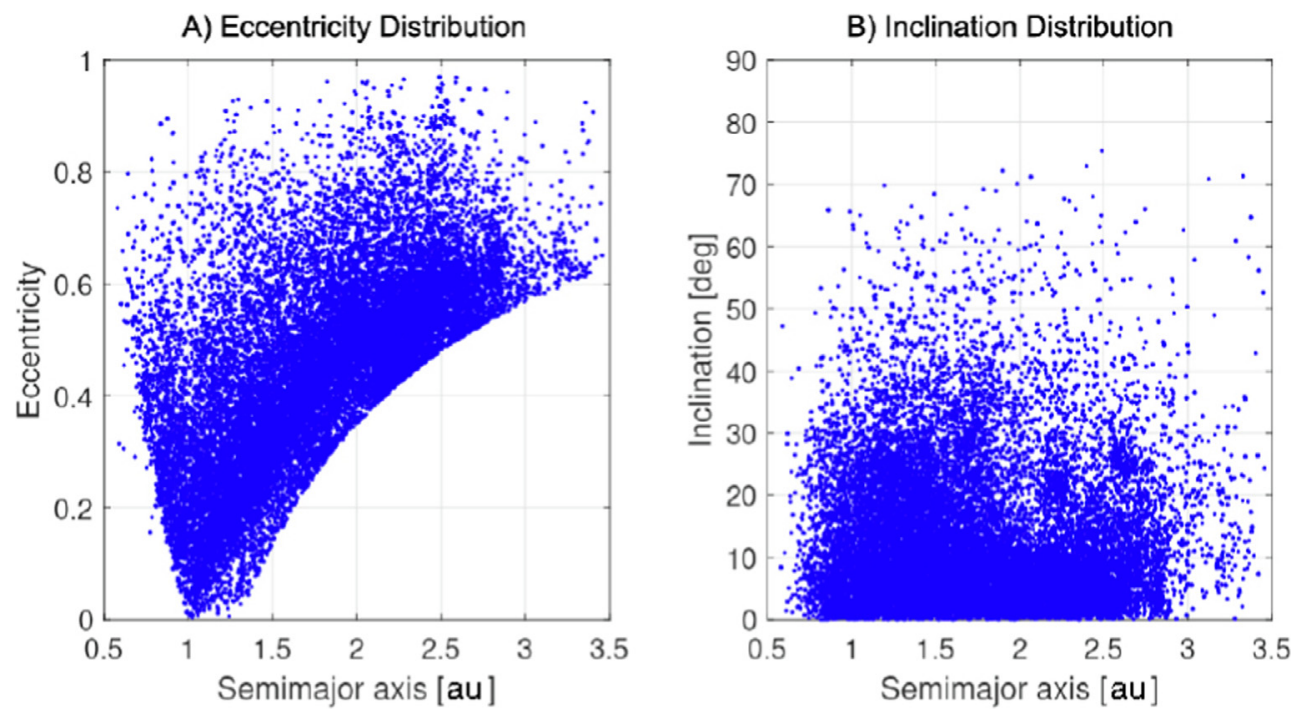

Fig. 4. Semi-major axis/eccentricity distribution (A) and semi-major axis/inclination distribution (B) of the considered NEO population.

of 2019. The diameter of the asteroids was estimated using to the following equation (Tedesco, 1994):

$D=\frac{1329}{\sqrt{p_{v}}} 10^{-0.2 H}$

where $D$ is the asteroid diameter in $\mathrm{km}, p_{v}$ is the albedo, and $H$ is the absolute magnitude. For asteroids whose albedo is not available, the value of $p_{v}=0.154$ was used corresponding to the average albedo of NEAs (Chesley et al., 2002). Fig. 5 shows the distribution of the estimated diameters of firstly all asteroids in the database, then only asteroids with an estimated diameter of less than $500 \mathrm{~m}$. It can be seen that the majority of asteroids have an estimated diameter of less than $100 \mathrm{~m}$.
The next step was to compute the Minimum Orbital Intersection Distance (MOID) (Gronchi, 2002; Gronchi, 2005) between all asteroids smaller than $500 \mathrm{~m}$, and a set of possible Initial Orbits (IOs) of the spacecraft. We define an IO as a solar orbit achieved post launch from the Earth and from which the tour can start, which may require an initial transfer after launch.

The aim was to identify the number of asteroids with a MOID lower than 0.01 au from each IO. IOs with low MOIDs are then further investigated to determine if a close flyby could be achieved by adjusting the trajectory.

The set of IOs was characterised by apoapsis and periapsis $\left(r_{a}\right.$ and $\left.r_{p}\right)$ in the range $[0.8,1.2] \mathrm{au}$, in steps of $0.05 \mathrm{au}$, and an inclination of 0 degrees with respect to the Ecliptic. 

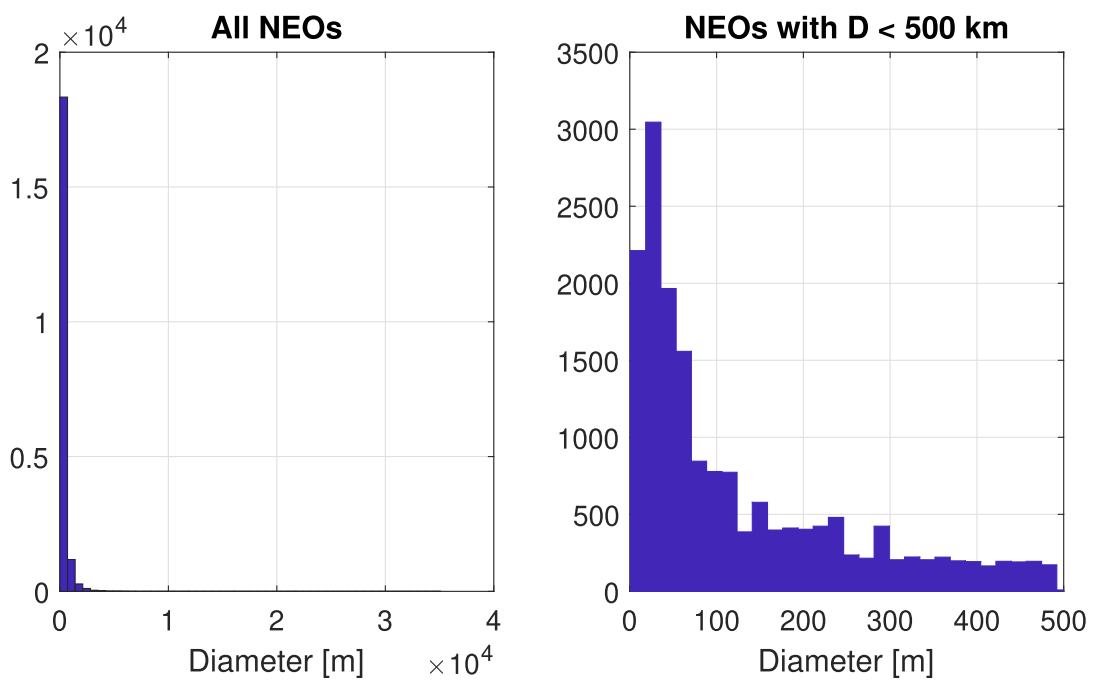

Fig. 5. Distribution of the diameter of the NEOs in the JPL database: all the NEOs in the database (left); only NEOs with diameter smaller than $500 \mathrm{~km}$ (right).

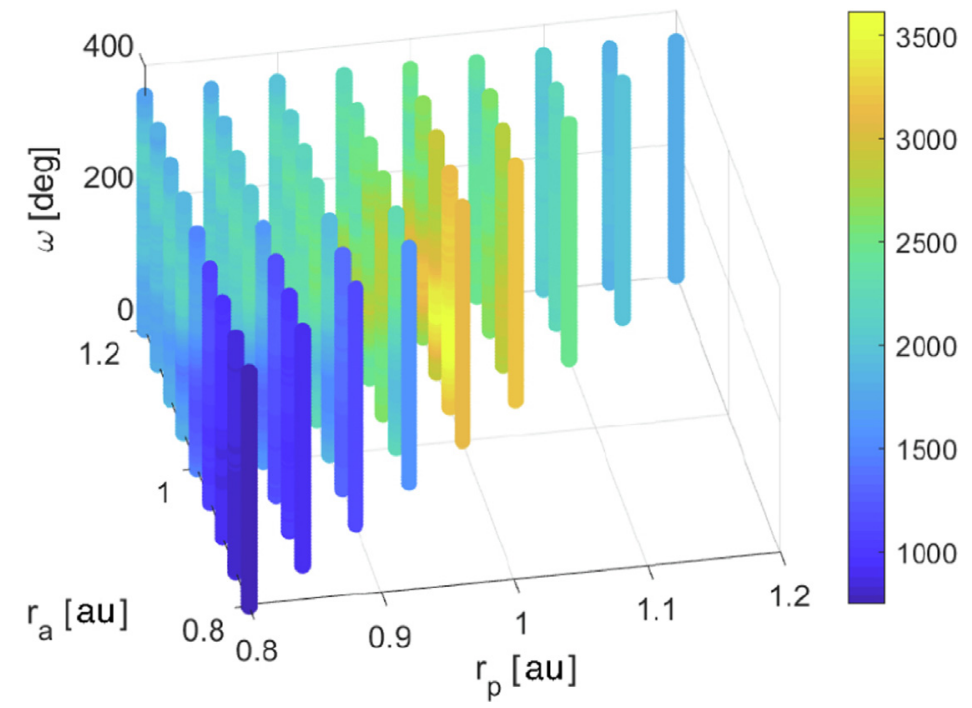

Fig. 6. Number of asteroids with MOID $<0.01$ au for different possible initial orbits of the spacecraft, characterised by different values of $r_{a}, r_{p}$ and $\omega$.

For non-circular orbits, the argument of the periapsis was allowed to vary from $0^{\circ}$ to $355^{\circ}$ in steps of $5^{\circ}$. The range of apoapsis and periapsis was chosen to ensure the IO remains relatively close to the orbit of the Earth, reducing propellant cost for the transfer to the IO.

This resulted in a set of 3240 possible IOs. The number of asteroids with a MOID $<0.01$ au from at least one orbit in this set is shown in Fig. 6 as a function of $r_{a}, r_{p}$ and $\omega$.

The selection was later restricted to the following combinations of $r_{a}$ and $r_{p}$ for which the number of asteroids with MOID $<0.01$ au was higher than 3000:

1. $r_{p}=r_{a}=1$ au

2. $r_{p}=1 \mathrm{au}, r_{a}=1.05 \mathrm{au}$

3. $r_{a}=r_{p}=1.05 \mathrm{au}$

4. $r_{p}=1 \mathrm{au}, r_{a}=1.1 \mathrm{au}$
Fig. 7 shows the number of asteroids with MOID $<0.01$ au for these four combinations, as a function of $\omega$. That is, the number of asteroids that we considered to be reachable for that IO as its argument of periapsis is varied.

The orbits in Fig. 7 are denoted as $\mathscr{O}_{i}$ with $i=1, \ldots, 4$. The next step was to define an initial mean anomaly for the IO at a given initial time (Carlo et al., 2018) such that the spacecraft and NEO were at the node of NEO's orbit roughly at the same time. We followed the same procedure as in Carlo et al. (2018) and determined that a flyby was indeed possible if the distance from NEO to spacecraft, when the NEO was at the node, was lower than $0.01 \mathrm{au}$. This phasing constraint further reduces the number of visitable asteroids. Given the uncertainty on NEO's ephemerides, imposing a tighter threshold at this stage would not help with the definition of the flyby. 


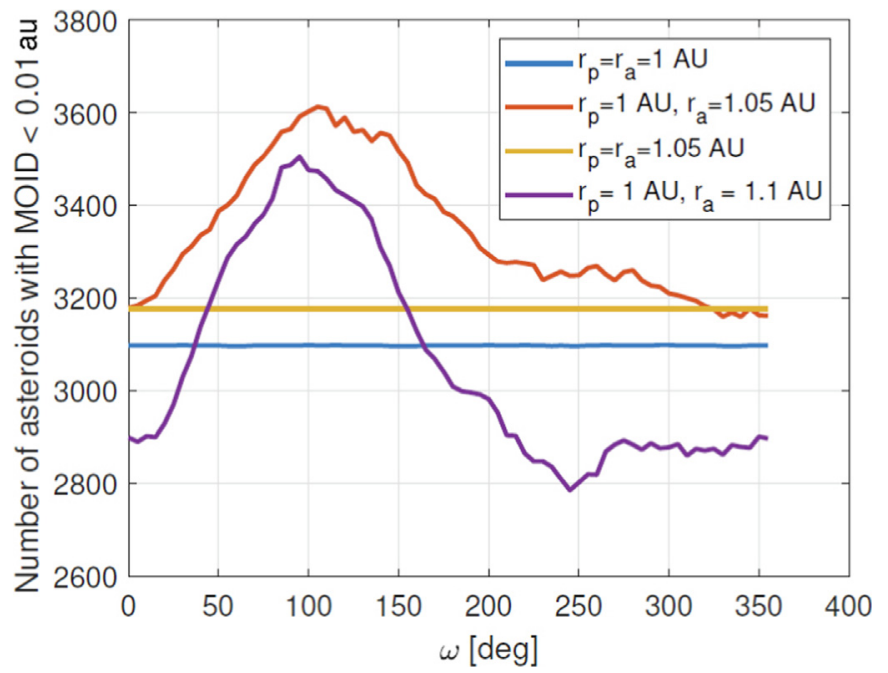

Fig. 7. Number of asteroids with MOID $<0.01$ au as a function of $\omega$, where MOID is measured with respect to 4 different IOs defined by 4 combinations of $r_{a}$ and $r_{p}$.

The phasing analysis was then followed by a tree-search using a Binary Tree (BT) (see (Carlo et al., 2018) for more details). For each IO, the BT selected only sequences that were possible with a total $\Delta V<2 \mathrm{~km} / \mathrm{s}$, and for which the relative velocity at all flybys was lower than $10 \mathrm{~km} / \mathrm{s}$. At this stage, the $\Delta V$ required to perform each flyby (that is, the $\Delta V$ required to go from distance $<0.01$ au, to distance equal to zero at the encounter), was estimated using a bi-impulsive Lambert transfer arc connecting two subsequent encounters, where an impulse is performed after each flyby to move the spacecraft to the next asteroid encounter. The impulsive model for the transfer was used as model for the BT because of the reduced computational time with respect to a low-thrust transfer model. However, the final reference tour was optimised for a low-thrust propulsion system.

The phasing analysis and sequence definition was performed for 24 initial dates on the orbits $\mathcal{O}_{i}$, for values of mean anomaly $M_{0}$ between $0^{\circ}$ and $359^{\circ}$. Fig. 8 shows the results of the BT for 12 different departure dates from the initial orbit in the year 2023 , for orbit $1\left(r_{p}=1 \mathrm{au}\right.$, $\left.r_{a}=1 \mathrm{au}\right)$. Each subplot reports the initial date that appears in the title of the subplot. The $x$ axis reports the total number of asteroids in the sequence; the $y$ axis reports the total $\Delta V$. Therefore, each dot corresponds to a possible mission, characterised by the number of encountered asteroids given on the $x$ axis and the $\Delta V$ reported on the $y$ axis. For each value on the $x$ axis, only the first 1000 solutions with the lowest $\Delta V$ are reported. Solutions for the remainder of 2023 for IO 1 and all of 2023 for the other three selected orbits are available in the appendix. The results in Fig. 8 are fairly typical compared with the other IOs and time periods.

It is important to stress that each solution in Fig. 8 is characterised by a specific value of $M_{0}$ for the spacecraft on the orbit at the defined IO departure date. At this stage, no consideration is made about the feasibility of reaching the selected IO, at the selected $M_{0}$, and at the selected date, in a given time of flight. It will be later shown that, in order to keep the transfer time lower than 2 years, not all the solutions in Fig. 8 can actually be reached.

As an example, Fig. 9 shows the $\Delta V$ required to transfer to IO number 4 , with a transfer trajectory that requires one full revolution around the Sun, for different values of $M_{0}(x$ axis) at different dates ( $y$ axis) in 2023. The figure shows that for some combinations of date and $M_{0}$, the transfer is not possible due to the required $\Delta V$.

From the binary tree and the $\Delta V$ plot for each IO, it was found that the departure date $30 / 11 / 2023$ for IO 4 is the
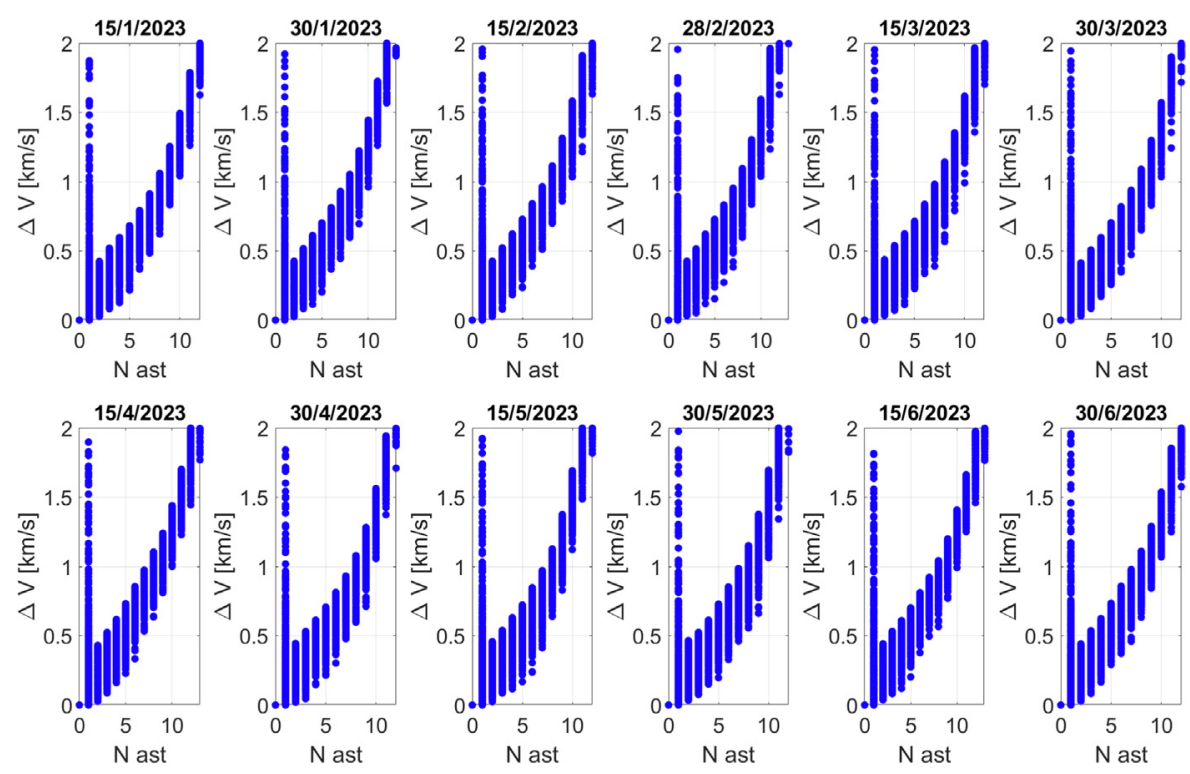

Fig. 8. Binary tree solutions for orbit $1\left(r_{p}=r_{a}=1 \mathrm{au}\right)$, for different departure dates, from January to June 2023 , at intervals of 15 days. Each subplot reports, for each number of visited asteroids, the top 1000 cheapest $\Delta V$ solutions. 


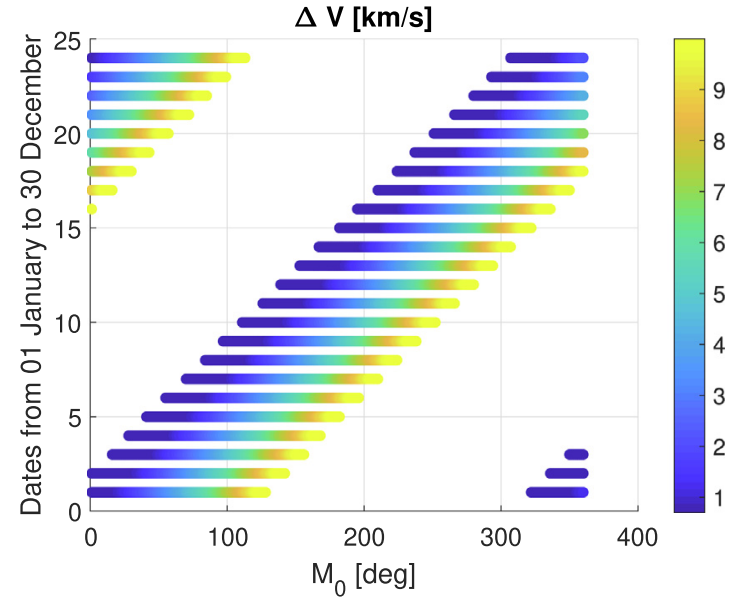

Fig. 9. $\Delta V$ required to transfer to IO number 4 , with a transfer trajectory that requires one full revolution, for different values of $M_{0}$ on IO number 4 ( $x$ axis) and for 24 different initial dates, at intervals of 15 days, in 2023 ( $y$ axis). The range of $\Delta V$ values is represented by the colorbar to the right of the figure. (For interpretation of the references to color in this figure legend, the reader is referred to the web version of this article.)

only case in which the number of asteroids in the sequence is as high as 15 . IO 4 is characterised by $r_{p}=1$ au, $r_{a}=1.1$ au and $\omega=95 \mathrm{deg}$. However, other combinations of orbital parameters, corresponding to different IOs, have resulted in sequences with more than 10 asteroids for every departure date in the required launch window. It should be noted that if a finer spacing in the orbital elements of the investigated set of IOs were used, more possible tours may have become apparent. To get a full set of all possible tours in a given departure window, finer spacings should be used and more IOs tested.

\subsection{Filter on asteroid size}

Most of the solutions that were found using only the launch date and $\Delta V$ as selection criteria had a large number of small asteroids. In the context of planetary defence, very small asteroids are less of a priority, hence we inserted another filter that removed all asteroids smaller than $50 \mathrm{~m}$ from the database before running the tree search. A further selection filter was included in the binary tree search to retain sequences with at least one asteroid larger than $150 \mathrm{~m}$. This further constraint was introduced to increase the scientific and planetary defence value of a tour. As it will be shown in the reminder of this paper, further considerations should include the visibility of the asteroid (its magnitude) in relation to the current knowledge of its ephemerides.

Figs. 10 and 11 show the solutions obtained on the reduced database of asteroids with diameter $D_{\text {ast }}>50 \mathrm{~m}$ and with start date of the tour in the year 2023. The $x$ axis shows the total number of asteroids in the sequence. The $y$ axis shows the number of asteroids for each sequence, with a diameter greater than $150 \mathrm{~m}$. For example, for the date $15 / 05 / 2023$, it can be seen that there are sequences with up to 4 total asteroids; the available sequences of 1,2 or 3 total asteroids have either no asteroid with diameter above $150 \mathrm{~m}$, or only 1 asteroid with diameter above $150 \mathrm{~m}$. On the contrary the sequence with 4 asteroids has two asteroids larger than $150 \mathrm{~m}$. It can be seen that the typical solution comprises 3-4 asteroids, one being larger than $150 \mathrm{~m}$. Solutions containing 5 objects with two larger than $150 \mathrm{~m}$ are available in 2023 , with start dates of June 30th and October 15th.

In order to choose a feasible tour one must match the initial value of $M_{0}$ on the orbit $\mathscr{O} \mathscr{E}$ and the departure date from the Earth. In principle, one can include a number of revolutions around the Sun to phase the departure from the Earth with the start of the tour. These phasing orbits have an impact on the total mission time and $\Delta V$. Hence we considered that a given sequence was feasible only if the phasing orbits did not require a significant increase in mission time and $\Delta V$.

\subsection{Reference solution}

By combining all considerations on mission time, minimum asteroid size and minimum $\Delta V$, the solution with

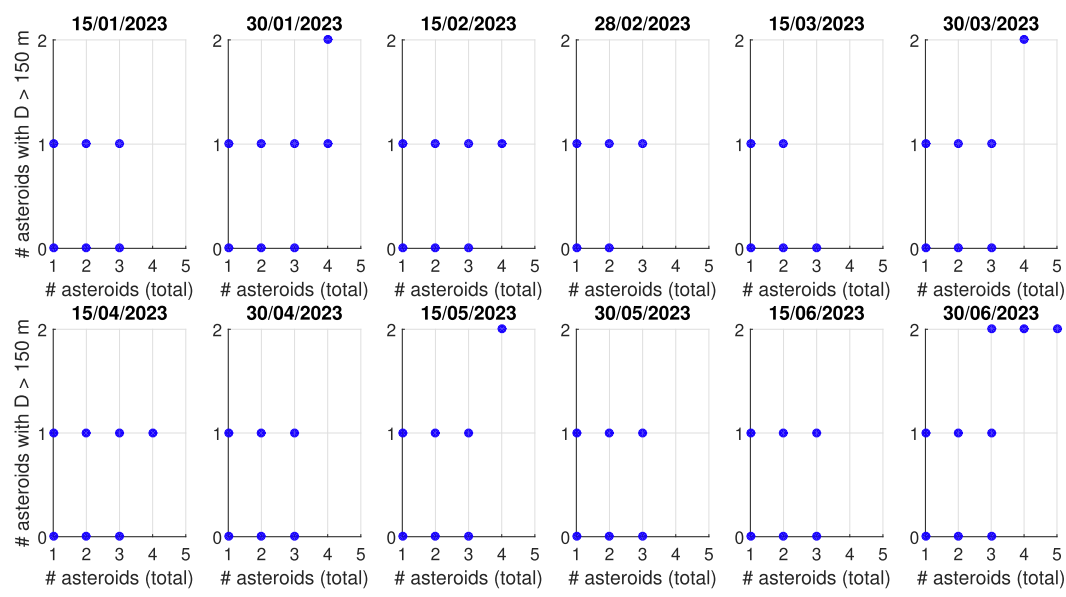

Fig. 10. Solutions with starting date of the tour from January to June 2023, at intervals of 15 days. Each subplot shows, for the identified sequences, the total number of asteroids in the sequence ( $x$ axis) and the number of asteroids with diameter greater than $150 \mathrm{~km}$ in the sequence $(y$ axis). 


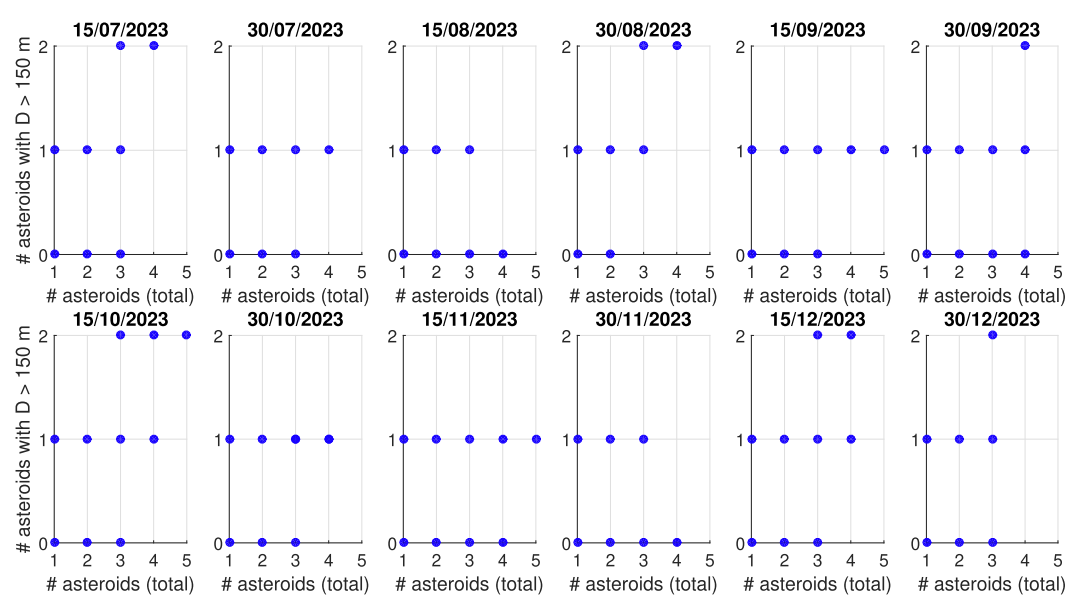

Fig. 11. Solutions with starting date of the tour from July to December 2023, at intervals of 15 days. Each subplot shows, for the identified sequences, the total number of asteroids in the sequence ( $x$ axis) and the number of asteroids with diameter greater than $150 \mathrm{~km}$ in the sequence ( $y$ axis).

Table 2

Details of the reference tour considered for this work.

\begin{tabular}{|c|c|}
\hline \multicolumn{2}{|c|}{ Transfer to $\mathscr{O} \mathscr{E}$} \\
\hline Launch date & 22 December 2022 \\
\hline Arrival date on orbit $\mathscr{O} \mathscr{E}$ & 30 January 2025 \\
\hline Transfer time to $\mathscr{O} \mathscr{E}$ & 2 years, 1 month \\
\hline$\Delta V$ to reach $\mathscr{O} \mathscr{E}[\mathrm{km} / \mathrm{s}]$ & 1.56 \\
\hline \multicolumn{2}{|c|}{ NEOs tour } \\
\hline Start date for the tour of NEOs & 30 January 2025 \\
\hline$M_{0}$ on $\mathscr{O} \mathscr{E}$ on 30 January $2025[\mathrm{deg}]$ & 325 \\
\hline Date of final flyby & 8 August 2027 \\
\hline Total tour time & 2 years and 7 months \\
\hline$\Delta V$ to realise the tour $[\mathrm{km} / \mathrm{s}]$ & 0.7 \\
\hline
\end{tabular}

starting date Jan 30th 2025, with 4 asteroid encounters, was chosen as the reference tour for the remainder of the study. The main characteristics of the reference tour can be found in Table 2. The table provides information about the lowthrust transfer from the Earth to the initial orbit of the spacecraft, $\mathscr{O} \mathscr{E}$, and about the tour of the asteroids.

Table 3 contains information on the asteroid in the sequence of flybys of the reference tour, including the dates of encounters, the estimated diameter of each asteroid, semi-major axis, eccentricity, inclination, and the relative velocities at each flyby. Figs. 12 and 13 show the lowthrust trajectories to realise the transfer from Earth to the orbit $\mathcal{O} \mathscr{E}$, and to perform the tour of the four asteroids. The low-thrust trajectory was optimised to obtain the thrust profile that provides the minimum $\Delta V$, using the toolbox FABLE (Fast Analytical Boundary-value Low-

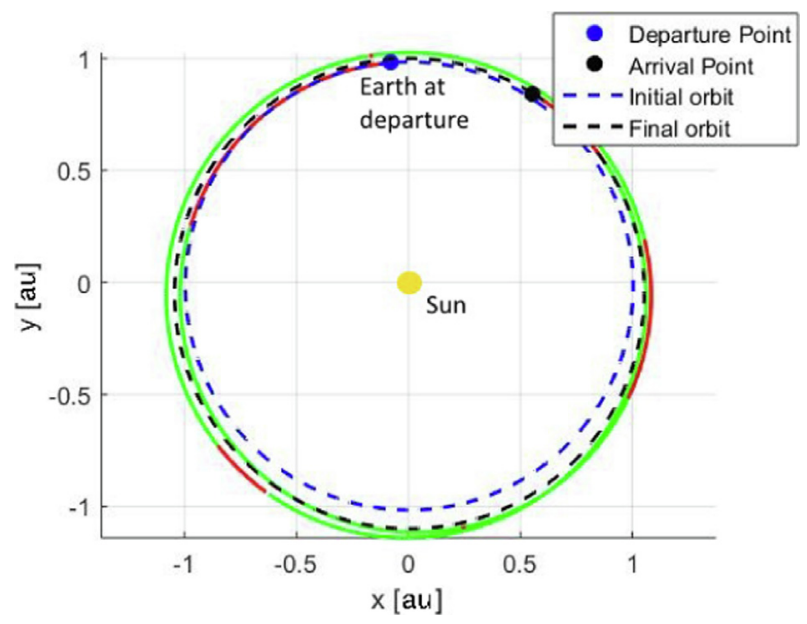

Fig. 12. Low-thrust transfer to $\mathscr{O} \mathscr{E}$.

thrust Estimator) (Carlo et al., 2018). The reference frame is inertial heliocentric with the $x$ axis pointing at the vernal equinox at epoch and the $z$ axis perpendicular to the ecliptic. In Fig. 12, thrust arcs are shown in red and coast arcs are shown in green. No difference in color is shown in Fig. 13 because the changes to the trajectory performed by the low-thrust engine are less pronounced in this case.

\section{Navigation requirements and trajectory corrections during the approach phase}

The orbital elements of the NEOs in the JPL database (JPL Small Body Database, 2019) are not known with full

Table 3

Information on the visited asteroids.

\begin{tabular}{lccccccc}
\hline SPK ID & Family & Date of encounter & Diameter $[\mathrm{m}]$ & $a[\mathrm{au}]$ & $\mathrm{e}$ & $i[\mathrm{deg}]$ & Flyby rel. vel. [km/s] \\
\hline 3723829 & Apollo & $1 / 10 / 2025$ & 71 & 1.09 & 0.18 & 14.8 & 8.91 \\
2508908 & Aten & $30 / 3 / 2026$ & 224 & 0.88 & 0.27 & 5.6 & 7.89 \\
2010302 & Amor & $1 / 11 / 2026$ & 467 & 1.27 & 0.14 & 4.37 & 3.06 \\
3802401 & Aten & $8 / 8 / 2027$ & 71 & 0.89 & 0.20 & 18.49 & 9.53 \\
\hline
\end{tabular}




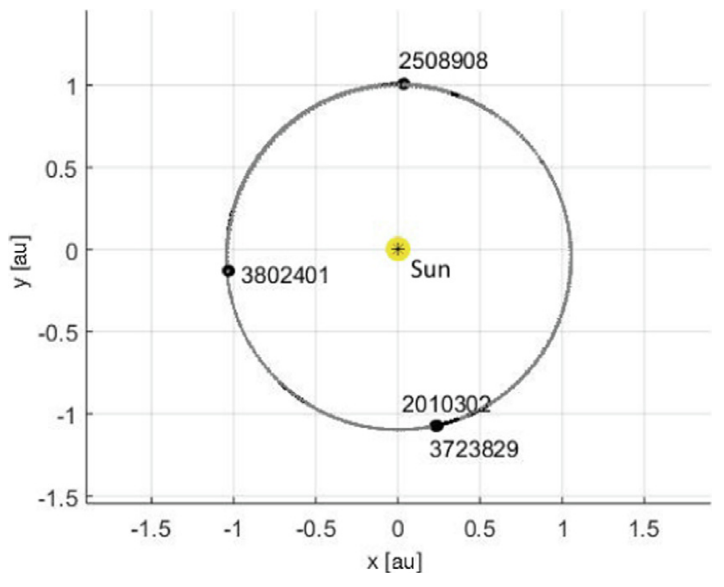

Fig. 13. Tour of the asteroids.

precision and have an attached uncertainty. This is due to the number and quality of the observations and is generally larger for smaller asteroids. Hence, the uncertainty in the orbital elements must be taken into account during the approach and flyby phases. Table 4 shows the 1- $\sigma$ uncertainty on the JPL orbital elements used for the trajectory design for the asteroids in the reference tour as reported in the JPL database.
In order to properly quantify the uncertainty in position and velocity of the asteroid at the time of each flyby the uncertainty on the ephemerides at the epoch in Table 4 was propagated to the expected time of the flyby. The resulting 3- $\sigma$ ellipsoid on the position components, centered on the nominal asteroid position, in the inertial ecliptic frame with the $x$ axis pointing toward the vernal equinox, is plotted for each asteroid in the reference tour in Fig. 14. As one can see in Fig. 14, the second and third asteroids in the sequence, whose ephemerides at the reference epoch are better known, have a rather low uncertainty at the flyby time, while the other two smaller asteroids present a more significant uncertainty. As this graph represents the 3- $\sigma$ ellipsoids, i.e. the ellipsoids enclosing 99.7\% of the probability associated with the possible positions of the asteroids, the points on their surfaces was taken as the worst-case displacement of the asteroid from its expected position.

\subsection{Optical navigation and orbit determination}

In order to ensure the required close flyby, deviations in the asteroid's true position from its expected position must be compensated. Due to the use of a low-thrust propulsion system, it should be ensured that the camera can acquire

Table 4

1- $\sigma$ uncertainty on the orbital elements of the visited asteroids (JPL Small Body Database, 2019).

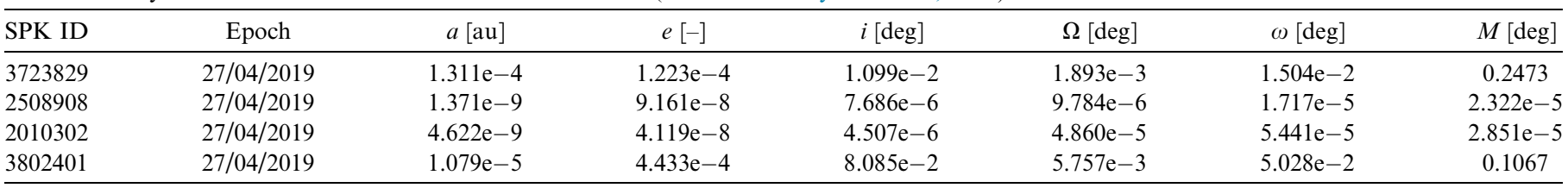
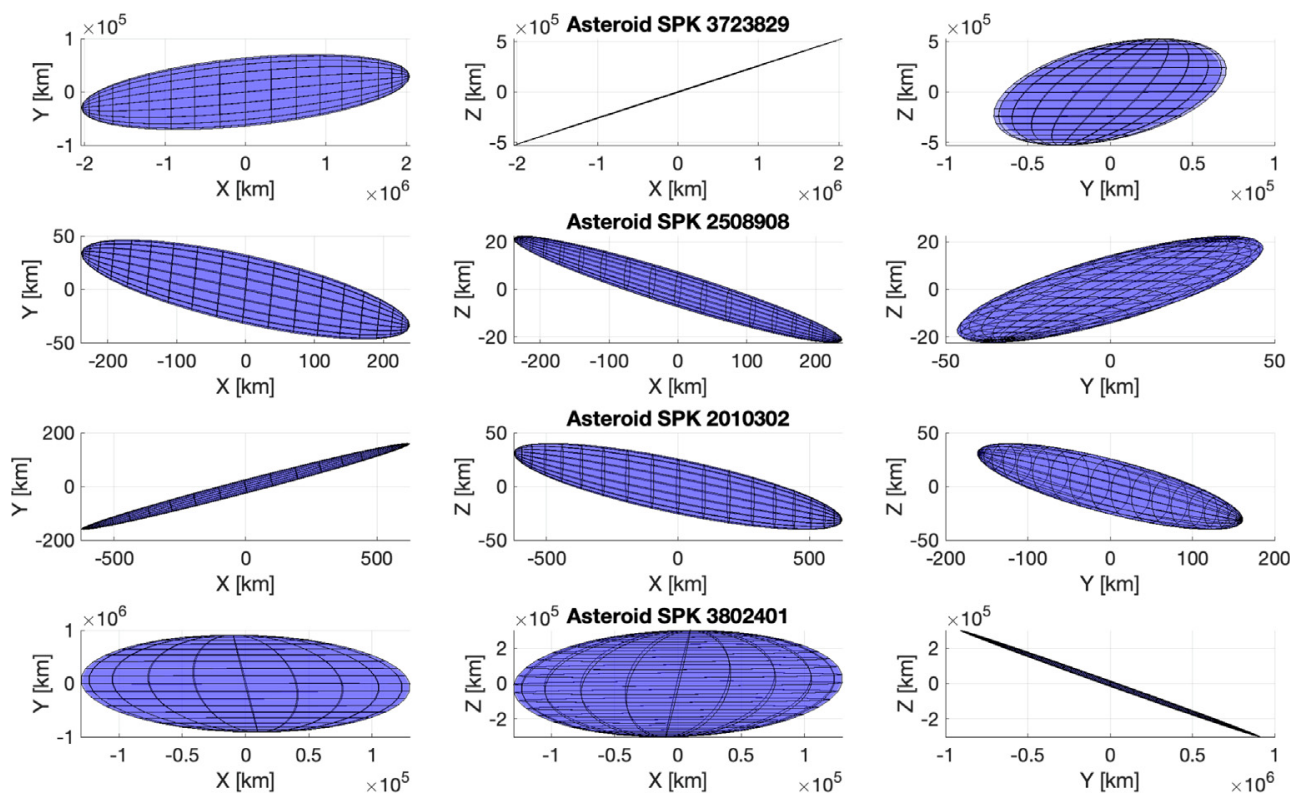

Fig. 14. Projection of 3- $\sigma$ ellipsoids for uncertainty in asteroid position at nominal flyby time centered in the nominal position. 
the object long enough in advance to allow time for lengthy TCMs to be performed. In theory, a long enough exposure would always allow the object to be observed, although the exposure time is limited by pointing stability causing image smearing, as well as pixel saturation from noise. This section analyses the exposure requirements and proposes a frame summation method to overcome the exposure limitations.

First, from orbital modelling, the distance to the target (first asteroid in the sequence, one of the smaller objects which are more challenging to image) as a function of time on approach is shown in Fig. 15, as well as the expected apparent magnitude at a given distance from the object. This can be used to determine the apparent magnitude as a function of time on approach.

By the definition of the magnitude scale, photon flux in a given wavelength band can be estimated from the apparent magnitude by comparison with a known flux for a different magnitude according to Eq. 2.

$m=-2.5 \log _{10}\left(\frac{F_{x}}{F_{x, 0}}\right)$

Here $m$ is the apparent magnitude of the object in question, $F_{x}$ is the flux from the source in wavelength band $x$, and $F_{x, 0}$ is the reference photon flux for that band at apparent magnitude zero (Bessell, 1979). To improve SNR, we assume a wide-band CCD which is sensitive to all wavelength bands between $U$ and I, summing the flux across each of these bands to obtain the total flux. For the purposes of photon counting for SNR estimation, the total flux is treated as having an average wavelength of $550 \mathrm{~nm}$.

This photon rate can now be used to compute an expected signal-to-noise ratio for a given exposure time. Here the SNR is defined by the common imaging definition (Schroeder, 1999)

$S N R=\frac{N_{p h}}{\sigma_{\text {noise }}}$
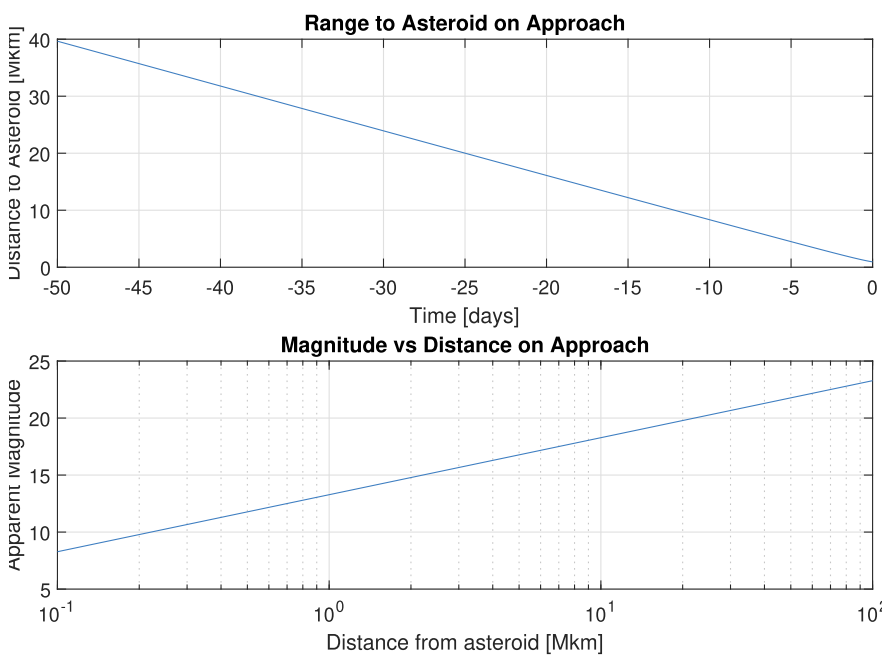

Fig. 15. Distance and apparent magnitude on approach for asteroid 3723829 , accounting for 90 degree phase correction. where $N_{p h}$ is the mean or expected number of signal photons collected per pixel in a given exposure, and $\sigma_{\text {noise }}=\sqrt{N_{\text {noise }}}$ is the standard deviation of the total noise, with $N_{\text {noise }}$ being the expected number of noise photons collected per background pixel in the same exposure.

Noise sources modelled included a mean read noise of 5 counts per pixel, and a dark count rate of $100 \mathrm{~Hz} / \mathrm{px}$. This dark noise rate is a pessimistic estimate due to the likely lack of large active cooling systems onboard a CubeSat if a small thermoelectric cooler and heat sink could be included in the camera design this would significantly reduce the dark count rate. Background from the sun is negligible as the camera will generally be used for OD pointed close to 90 degrees from the ecliptic plane, so inclusion of a small baffle would preclude sunlight from the image.

Light from background stars should also be included in the noise estimation. To approximate the number of stars of a given magnitude in a given angular area of sky, the total number of stars of a given magnitude range in the sky (obtained from the (HNSKYPlanetarium Program, 2020) which uses data from the Tycho-2 and UCAC4 star catalogs) divided by the total angular area of a sphere gives the average angular areal density for the magnitude band. This data had to be extrapolated to fainter magnitudes, as these catalogs only contain stars brighter than magnitude 16.

For a given magnitude band to be considered as part of an approximately uniform background, star density of the band must be high enough that stars are not significantly separated. The condition was imposed that if the average angular separation per star is less than half of the point spread function (PSF) width, the PSF would blur the stars together into an approximately uniform background. It was found that for the given camera, only stars dimmer than magnitude 17 are dense enough to form part of this quasi-uniform background. The same method for calculating total U-I band flux was used to for these background stars as for the signal flux to give a total count rate per pixel from this background source. Stars down to magnitude 25 were included in the background count rate calculation, which result in a mean photon rate of approximately $40 \mathrm{~Hz} / \mathrm{px}$.

It should be noted that there do exist brighter stars of lower density than are included in this background. Although the SNR analysis does not account for these, they may happen to coincide with the target at less than one PSF distance. In this case, these known stars would be brighter than expected and the presence of the target may be able to be inferred from this. For the brightest stars, it is assumed that the timing of the observation would be such that the expected position of the object would be far enough away from these stars as to avoid coincidence. It should also be noted that in reality the density of stars in the sky varies with direction - with a higher density in the plane of the Milky Way. Although the exact 
direction of camera OD observations is not known at this stage, and will vary with different tours, it is known that they will generally be close to 90 degrees to the ecliptic since the targets are inclined. Since the planes of the Milky Way and ecliptic are at approximately 63 degrees from one another, it can be assumed that with this camera's 5 degree FOV, observations will not be in the direction of the Milky Way, so the above average star density of that region is not expected to be inside the FOV.

The PSF of the camera was modelled as a uniform spread over 5 pixels. At these distances, the angular size of a 71 metre asteroid would be significantly less than one pixel and can thus be treated as a point source spread by the PSF.

Fig. 16 shows the minimum required exposure at a given time before flyby to achieve an SNR of at least 1.5 and 3 to allow the target to be acquired. SNR of 3 is a more conservative estimate, while SNR 1.5 is the same threshold used for NEA Scout (Lightholder et al., 2019).

wIn reality, the maximum duration of a single exposure would be limited by both pointing stability and pixel saturation. Frame addition is a common technique used to create large effective exposures in astronomy (White et al., 2007; Gruen et al., 2014) which could be applied to this scenario to overcome these limiting factors. Although it has never been used in the context of asteroid navigation, Lightholder et al. (2019) has proposed the technique for use in the future NEA Scout mission. This would require a detailed characterization of the jitter characteristics of the ADCS unit installed in the final spacecraft to identify typical jitter slew rates and peak-to-peak magnitudes over the course of a given duration of sub-exposure. The XACT-15 CubeSat ADCS unit from Blue Canyon Technologies for example is capable was measured in-orbit aboard a $3 \mathrm{U}$ CubeSat by Mason et al. (2017), and was found to have a peak-topeak pointing stability of $0.0072^{\circ}$ over a $10 \mathrm{~s}$ period (i.e. $0.0036^{\circ}$ maximum absolute deviation from the desired direction) using this unit. This is less than the pixel size in the proposed camera, although longer timescales were not reported so there is no guarantee that the same performance could be maintained over a longer single exposure, as well as the question of how the unit would perform in a larger CubeSat.

\subsection{Trajectory correction maneuvers}

Correction maneuvers must be performed to meet the desired flyby altitude for mass estimation. These corrections are planned to compensate deviations in the spacecraft trajectory and uncertainty on the asteroid ephemeris. Re-targeting starts once the two spacecraft have acquired the asteroid and updated its ephemerides. Therefore, only the uncertainty in the relative state between the spacecraft and the asteroid is considered in this preliminary work.

The main TCMs are performed using a high-thrust secondary propulsion system. Using the primary low-thrust engine would require long thrust arcs before the flyby, which would limit critically the operational time and resources of other systems. The selected secondary propulsion system is composed of two Aerojet Rocketdyne MPS130-1U (MPS-130 Innovative Propulsion Solutions for SmallSats, 2020) (nominal thrust $1.25 \mathrm{~N}$ ) mounted at different corners of the spacecraft. Although part of the propellant will serve partially for ADCS, e.g. for reaction wheels desaturation, the total budget for TCMs is conservatively estimated as $\Delta V \approx 110 \mathrm{~m} / \mathrm{s}$. Fig. 17 shows the reachable deviations around the nominal trajectory that are achievable by using the high-thrust propulsion system in a given time constraint - i.e. if the orbit of the asteroid is updated $n$ days in advance, only $n$ days are available to perform the trajectory correction maneuver. For $n$ days, a single point in the corresponding reachable set is computed by propagating the spacecraft from $n$ days before flyby with an initial impulsive maneuver and computing the deviation from the nominal state. Then, the whole reachable set is constructed by systematically varying the thrust directions and magnitude, up to the maximum allowed value.

By comparing this reachable set with Fig. 14, the minimum time before flyby to start the orbit determination and correction campaign can be assessed. For the second and third flyby in the sequence, the expected deviations are so small, because of very accurate initial ephemerides, that they can be corrected in few days. For the first and last asteroids in the sequence, the worst-case deviations are larger than the reachable set even when the orbit determination and correction phase starts almost one month before the flyby.

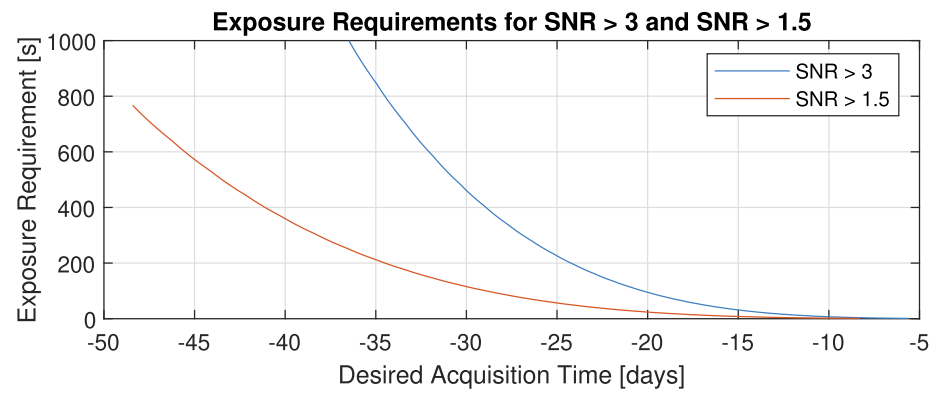

Fig. 16. Minimum exposure requirement at a given time before flyby to achieve SNR $>1.5$ and $\mathrm{SNR}>3$. 

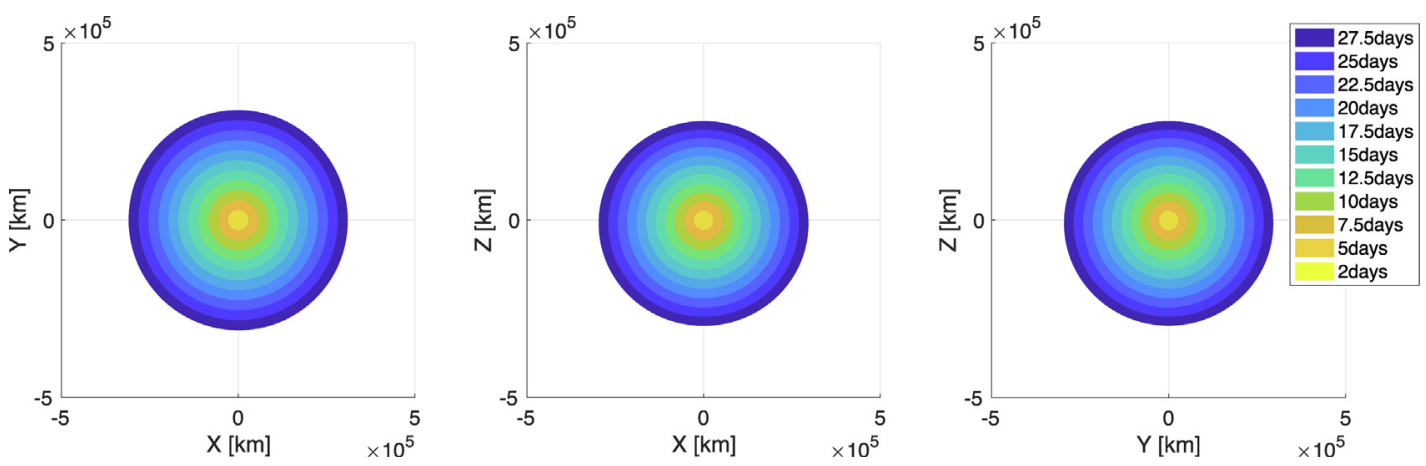

Fig. 17. Set of deviations which are possible to compensate with secondary high-thrust propulsion for different colour coded time intervals for the first flyby in the reference sequence. (For interpretation of the references to color in this figure legend, the reader is referred to the web version of this article.)
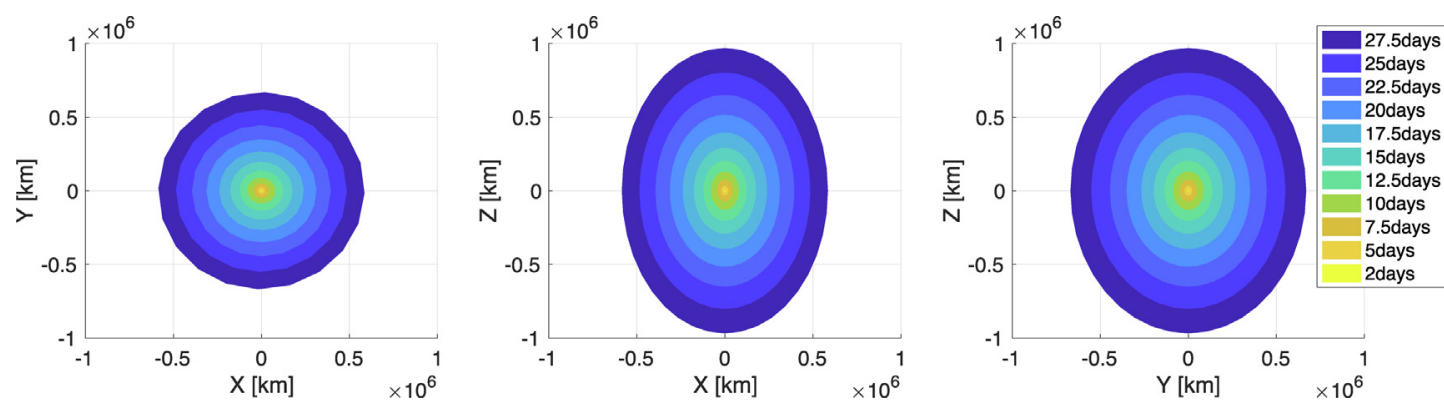

Fig. 18. Set of deviations which are possible to compensate with primary low-thrust propulsion for different colour coded time intervals for the first flyby in the reference sequence. (For interpretation of the references to color in this figure legend, the reader is referred to the web version of this article.)

In the contingency of deviations which cannot be compensated by the high-thrust engines, the reachable set could be further expanded by using the primary low-thrust engine. Fig. 18 shows the reachable set for the low-thrust engine. For $n$ days, a single point in the corresponding reachable set is computed by propagating the spacecraft from $n$ days before flyby with a control employing maximum thrust in a fixed direction and computing the deviation from the nominal state. In this case, the set is built by varying the thrust direction in this simple guidance law. This set is larger than the high-thrust set due to the small high-thrust $\Delta V$ capability and therefore can be used when the deviations cannot be compensated with the secondary engine. Effectively, the low-thrust reachable set is only time-limited due to the large propellant reserve, while the high-thrust set is both time- and propellant-limited, with the maneuver being performed earlier still providing a compounding effect.

The $\Delta V$ required by the low-thrust correction maneuvers for the asteroid sequence considered is considerably lower than both the nominal $\Delta V$ to realise the tour (see Table 2) and the high-thrust $\Delta V$ budget in all instances because of the relatively short thrusting arcs and highly efficient electric engine. However, using the primary engine involves long thrusting arcs and therefore lowers considerably the operational time that can be allocated to other systems pre-flyby due to the engine pointing direction requirement and power draw precluding the use of commu- nications systems and further camera observations during the burn.

Furthermore, in the final approach phase, the GNC system acquires more accurate data about the spacecraftasteroid relative position and precise maneuvers should be performed. The low-thrust system is generally unable to perform precise re-targeting in a short time window because of its limited control authority. Hence, the final TCM phase is achieved by means of the high thrust propulsion even in the case of low-thrust large TCMs. Hence, a mixed high- and low-thrust strategy could be required for precise flybys. This more complex guidance is not investigated further in this paper and it will be the object of a dedicated study for the NEACORE's GNC system.

In the case that the deviation still exceeds the reachable set of the engines, the corresponding asteroid cannot have its mass estimated and the science return is limited only to improved knowledge of its orbit.

\section{Scientific measurement phase}

The measurement phase of the mission $\left(-T_{c 1}\right.$ to $+T_{c 2}$ in Fig. 2) proceeds as follows. Before flyby and after TCMs have been completed (around 1 day before the moment of flyby), the two satellites will begin monitoring their relative separation using their LIDARs. On final approach to the asteroid, the spacecraft align themselves such that the asteroid will pass through the field of view of the camera 
and the path of the LIDAR pulses. Because of the extremely high relative velocity, the spacecraft cannot rotate quickly enough to track the asteroid. The angular velocity of the asteroid in the sky during a $9.53 \mathrm{~km} / \mathrm{s}$ flyby at $10 \mathrm{~km}$ peaks at $953 \mathrm{mrad} / \mathrm{s}$. We excluded the use of movable periscope-style optics as this would increase size, weight and power requirements, complexity and fast rotation of heavy optics would induce significant torque on the spacecraft.

As the object passes in front of the camera, images are captured together with altimetric measurements of the asteroid's surface from the LIDAR as the two instruments have aligned pointing vectors. The $3 \mathrm{D}$ shape will be reconstructed from these camera observations at different angles. Combined with range measurements from the LIDAR this will give the physical size of the asteroid and a precise measurement of the flyby altitude. Additionally, variations in the range as the asteroid travels through the beam path will allow some limited reconstruction of topology along the beam axis, as shown in Fig. 22. After the flyby, the spacecraft will resume inter-satellite ranging (ISR) measurements with their LIDARs, and determine the perturbed trajectory due to the asteroid's gravity field over the next 5 days.

A model was developed to assess the maximum range at which the LIDAR could detect a returning pulse from an asteroid, and was further adapted to the ISR case, to assess the accuracy with which relative measurements and hence asteroid masses could be measured. First the basic model for direct asteroid ranging will be described, then the modifications for ISR measurements and how this is used for mass estimation will be presented along with test cases and an assessment of the predicted performance of the measurement strategy from the ISR model.

In the case of a dual flyby, the LIDAR is not actually required to range the asteroid directly in order to determine its physical size or the flyby altitude. Knowledge of the separation of the two spacecraft, camera pointing vectors, and the angular size of the asteroid observed by the cameras allows the size of the asteroid to be calculated geometrically. However, having a LIDAR powerful enough for direct ranging of the asteroid still allows for the platform to be used in single-flyby scenarios, or allows for size to be estimated should one spacecraft of a pair be lost in transfer, so knowledge of the requirements of the LIDAR in these scenarios is important.

\subsection{LIDAR model for asteroid ranging}

Luo et al. (2015) modelled the rate of primary electron generation in a single photon avalanche photodiode (SPAD) based detector to assess performance limitations, which forms the basis for this model. The initial derivation follows closely to that of Luo, and the rate of primary electron generation per pixel due to signal photons as a function of time was derived to be
$S_{P E}(t)=\frac{\eta_{q} \eta_{t} \eta_{r} p_{v} F_{f i l l} F_{r e f} F_{c o l}}{h v n_{p x}} P_{e m i t}\left(t-\frac{2 R}{c}\right)$

where $\eta_{q}$ is the detector quantum efficiency, $\eta_{t}$ and $\eta_{r}$ are the optical transmission and receiving efficiencies, $p_{v}$ is the target albedo, $F_{\text {fill }}, F_{\text {ref }}$ and $F_{c o l}$ are respectively the SPAD array fill factor (including microlens array fill factor), the fraction of outgoing light that hits the target object, and fraction of reflected light that is collected, $h v$ is the photon energy and $n_{p x}$ is the number of pixels in the modelled array. $P_{\text {emit }}(t)$ is the emitted laser power as a function of time (modelled to have a temporally square profile with pulsewidth $100 \mathrm{ps}$, amplitude scaled to the pulse energy), $R$ is the range to the target and $c$ is the speed of light. $p_{v}=0.154$ is assumed for asteroid targets.

Due to beam divergence, the beam area at the target will be much larger than it was when the pulse was fired, with divergence half angle equal of $\theta=M^{2} \frac{\lambda}{\pi w_{0}}$ (Duarte, 2017), where $M^{2}$ is the beam quality factor (set equal to 1.3 in this paper), $\lambda$ is the signal wavelength and $w_{0}$ is the waist radius of the beam, taken to be equal to the radius of the emitter. The target is assumed to reflect isotropically across a hemisphere with radius equal to the range from target to satellite. $F_{r e f}$ and $F_{c o l}$ hence are defined as follows

$F_{\text {ref }}=\min \left(1, \frac{A_{\text {ast }}}{A_{\text {beam }}}\right)$

$F_{c o l}=\frac{A_{c o l}}{2 \pi R}$

where $A_{c o l}$ is the area of the collecting optic, $A_{\text {beam }}$ is the area of the spot size at the target, and $A_{\text {ast }}$ is the apparent area of the asteroid from the spacecraft's perspective. The total rate of primary electron generation per pixel is

$T_{P E}(t)=\frac{1}{2}\left(S_{P E}(t)+N_{P E}(t)\right)+D C R$

where $N_{P E}(t)$ is the noise primary electron (PE) generation rate, derived similarly to the above and accounting for both direct solar photons and solar photons reflected from the target, $D C R$ is the per-pixel dark count rate, and the factor of $\frac{1}{2}$ is due to the signal being split over two identical SPAD arrays as previously mentioned.

Photon counting is a probabilistic process and follows Poisson statistics. As in Luo et al. (2015), the probability of detecting $\mathrm{k}$ photons in a given time window is given by

$P(k)=\frac{K_{a v}^{k}}{k !} e^{-K_{a v}}$

where $K_{a v}$ is the average number of photons expected in the time window. Setting $k=0$ gives the no-trigger probability for a given time bin, and $1-P_{\text {notrigger }}$ is the probability that the pixel will trigger. Thus, assuming $T_{P E}(t)$ is constant across one time bin,

$P_{\text {trigger }}=\left(1-e^{-T_{P E}(t) t_{b i n}}\right) P_{\text {untriggered }}$ 
where $t_{b i n}$ is the duration of one time bin and $P_{\text {untriggered }}$ is the probability that the pixel has not already been triggered since the start of the range gate. This is set to $10 \mathrm{ps}$ in these simulations, so that the spike in the histogram spans multiple time bins. The probability for that pixel having not triggered in all previous time bins since the beginning of the range gate is

$P_{\text {untriggered }}=\prod_{i=1}^{n-1} e^{-T_{P E}\left(t_{i}\right) t_{\text {bin }}}$

where $t_{i}$ is the start time of each time bin relative to the gate start time, $n$ is the time bin in question, and taking the product over $i$ accounts for all previous time bins since the start of the range gate. Thus Eq. (9) becomes

$P_{\text {trigger }}=\left(1-e^{-T_{P E}(t) t_{\text {bin }}}\right) \prod_{i}^{i-1} e^{-T_{P E}\left(t_{i}\right) t_{b i n}}$

Per-pixel trigger probabilities are multiplied by $n_{p x}$ to determine the mean number of pixels triggered in each time bin, which is then used as the mean of a Poisson distribution to generate a random number which is taken as the actual number of triggers in that time bin. A histogram is plotted, giving a simulated measurement of a returning laser pulse. An example measurement histogram can be seen in Fig. 19. The data is smoothed by conversion to a rolling average across $\pm t_{\text {pulse }} / 2$ in order to improve the curve fitting for spike detection.

\subsubsection{Noise sources}

A number of noise sources were included in the model to more realistically simulate measurements. Dark count rate (DCR) - the rate of spontaneous pair production in an unilluminated photodiode - was modelled as $50 \mathrm{kHz}$ per pixel. This is likely a pessimistic estimate, as DCR in InGaAs SPADs varies widely in the literature (Itzler et al., 2007; Itzler et al., 2011) and is also temperature dependent, with cooler detectors exhibiting lower DCR. Itzler et al. (2011) characterised a $32 \times 32 \mathrm{InGaAs}$ SPAD array similar to the array that is modelled in this paper, and all pixels had a DCR of less than $50 \mathrm{kHz}$. Thus this is a reasonable estimate for initial performance modelling.

Background signal-wavelength photons from the Sun were included as an additional noise source. This is modelled as having a spectral irradiance equal to that of sunlight at the signal wavelength of $1064 \mathrm{~nm}$ and at a distance of 1 au from the Sun, which is $0.647 \mathrm{~W} \mathrm{~m}^{-2} \mathrm{~nm}^{-1}$ (Sun et al., 2006). A bandpass filter in the optical path of $\pm 2.5 \mathrm{~nm}$ around the signal wavelength was assumed. The worst case scenario for sunlight noise was modelled, with the collector being exposed to normally incident sunlight. Solar photons reflected from the target object and impinging on the collector were also accounted for. Full illumination of the target with sunlight was also assumed for the worst case reflectance noise.

In asteroid ranging, for each pulse a surface slope angle was randomly generated between 0 and 60 degrees which spreads the pulse temporally. Since the range is detected by centroid of a fitted Gaussian curve, this will widen the effective pulse width and introduce uncertainty in the detected range.

\subsubsection{Detection criteria}

A simple algorithm was developed to detect spikes in the produced histograms. A 4-term Gaussian was fitted for each, and the term with the highest amplitude was selected as the 'detection' for that simulation. Fit coefficients were bound such that the centroid must lie within the detection window, and the width must be less than 10 pulsewidths. A minimum threshold must be set for the amplitude of the fitted curve to be considered a signal spike and not a noise fluctuation. This was derived by observing the behaviour of the model when only background counts were modelled. A total of 500 dark runs $\left(E_{\text {pulse }}=0\right)$ of the model were simulated to determine the distribution of fitted spike amplitude, and a value of 5 standard deviations above the mean was used as the threshold for the fit to be considered a true detection in subsequent simulations.
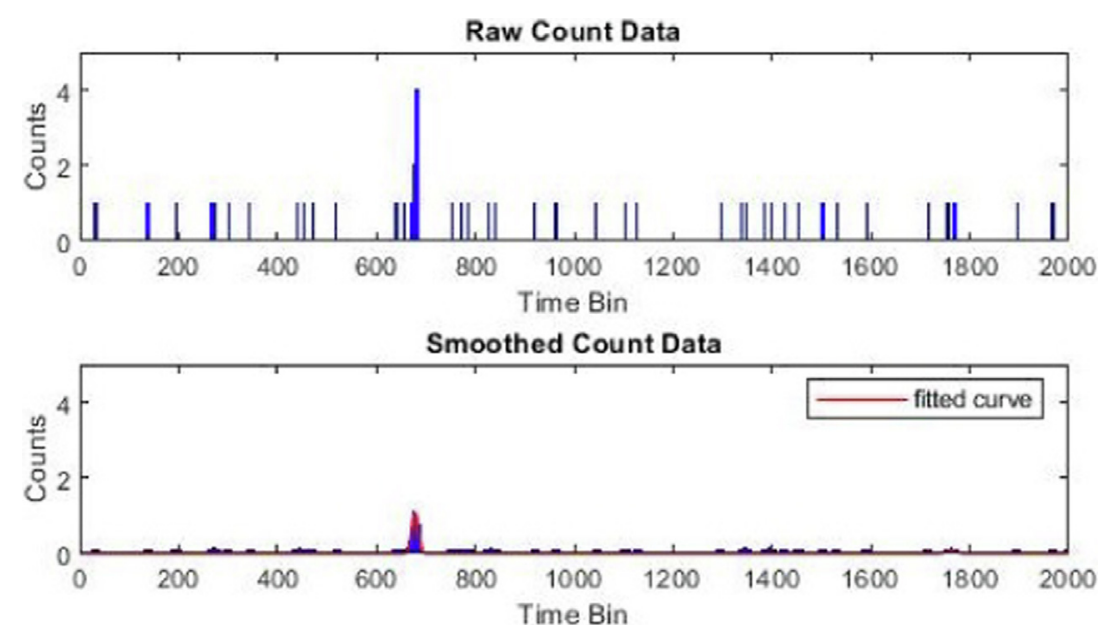

Fig. 19. Simulated measurement of a single range gate with fitted Gaussian curve. 


\subsection{LIDAR model for inter-satellite ranging}

In inter-satellite ranging, a two-way ranging approach is assumed, where both satellites point their LIDARs at each other. When satellite A fires a pulse, it is detected by satellite $\mathrm{B}$, which in turn fires a response pulse back at satellite A. This results in a much stronger detected pulse at satellite A than the case of a simple one-way LIDAR detecting a pulse scattered from satellite $B$, while also nullifying temporal pulse distortion as a satellite is not a flat plane. Thus this technique can be employed at much longer ranges using significantly lower pulse energy than if simple scattering of the primary pulse was used to detect range. In fact, a pulse energy of only $1 \mu \mathrm{J}$ was used in simulations, which was still easily detectable at ranges of $100 \mathrm{~km}$, significantly more than would actually be required of the system, as it will be shown later than extremely close flybys are required for this technique to be feasible for low-mass asteroids.

The rate of signal PE generation is defined similarly to the asteroid case, with the removal of the factors $F_{\text {ref }}$ and $p_{v}$, and with $F_{c o l}$ that is no longer defined as a fraction of a hemisphere, but as a fraction of the beam area collected by satellite A, fired by satellite B. Additional time delay factors, $t_{\text {response }}$ and $t_{\text {jitter }}$ should also be added, such that

$S_{p e}^{I S R}(t)=\frac{\eta_{q} \eta_{r} F_{\text {fill }} F_{c o l}}{h v n_{p x}} P_{\text {emit }}\left(t-\frac{2 R}{c}-t_{\text {response }}-t_{j i t t e r}\right)$

$t_{\text {response }}$ is an additional time delay that accounts for the time it takes to process an incoming pulse and generate a return signal. $t_{j i t t e r}$ is a noise term due to variations in $t_{\text {response }}$ caused by the computer clock on the responding satellite. A new value is generated for each simulated pulse and added to the return signal delay. $t_{\text {jitter }}$ is a normally distributed randomly generated time offset with a mean of 0 $\mathrm{s}$ and a standard deviation of $5 \mathrm{ps}$.

Beam divergence is assumed to be significantly larger than in the asteroid case in order to reduce the pointing accuracy requirement on the LIDAR due to the small angular size of a spacecraft compared with that of an asteroid, and reduce the likelihood of the spacecraft drifting out of each other's laser beams. It should be mentioned that we assume lateral position drift of the other spacecraft will be monitored using a long-exposure setting on the camera which should be sensitive to the wavelength of the LIDAR - and tracking the bright spot of the incoming pulses as they move slowly across pixels. This would allow for attitude adjustments to be performed to keep the satellites centered in each other's FoV, ensuring that small range offsets are not introduced by misaligned pointing vectors.

Due to the incredibly sensitive nature of single photon counting detectors, it was found that for the two-way ranging approach, even with $R=100 \mathrm{~km}$ and high beam divergence, very low pulse energies on the order of $1 \mu J$ are sufficient to be detected. Even a weak $1 \mu \mathrm{J}$ pulse at $1064 \mathrm{~nm}$ consists of $5.4 \times 10^{12}$ photons - spread over a $10 \mathrm{~km}$ circular spot, there are $6.8 \times 10^{4}$ photons $\mathrm{m}^{-2}$, and approximately 350 impinge on the $8 \mathrm{~cm}$ diameter circular aperture. Since a LIDAR designed to detect scatter from an as asteroid at similar distances as will be required for ISR, a hugely overpowered laser for this application would already be on board the spacecraft. So it can be assumed that any practical limit on range for ISR via the two-way ranging method is well beyond the actual ranges that are required.

With $1 \mu \mathrm{J}$ pulse energy, 100 ps square pulse width, and 5 ps mean jitter timing offset, 500 ISR measurements were simulated to determine the expected noise characteristics of ISR measurements for use in modeling later in the paper. The distribution around the mean can be seen in Fig. 20. The measurements follow an approximately normal distribution with a standard deviation of $1.13 \mathrm{~mm}$.

\subsection{Numerical modelling}

This section collects the results of two sets of simulations. One set tests the capability of the LIDAR to measure the range between spacecraft and asteroid during close approach and flyby. The second set of simulations is to assess the intersatellite measurement capability of the LIDAR. The latter is very important to define the requirements of the gravimetric experiment.

\subsubsection{Asteroid Ranging}

In order to determine the maximum reliable operating range of a LIDAR in this mission scenario, and hence the required laser pulse energy, the model described in Section 5.1 was used to simulate 200 pulses for each of a set of combinations of $E_{\text {pulse }}$ and $R$. $E_{\text {pulse }}$ was tested between $125 \mu \mathrm{J}$ and $1.5 \mathrm{~mJ}$, and $R$ between $10 \mathrm{~km}$ and $150 \mathrm{~km}$. Each combination produces a distribution of detected signal spike amplitude which must be assessed to determine whether this is detectable or not. The threshold for a detectable signal amplitude was determined by running 'dark' simulations where $E_{\text {pulse }}=0$ and analyzing the noise fluctuations. The largest fitted spike in each dark simulation was assumed to be a detection in that case, and the distribution of spike amplitudes was found for 1000 dark runs. The threshold was set at 3 standard deviations above the mean spike amplitude, and this value used as the minimum required spike amplitude to register a detected returning pulse.

An acceptable detection probability must also be set, however, in this work, this was not determined in detail. Fig. 21 shows the maximum operational range of LIDARs of various pulse energies with detection probabilities varying from $50 \%$ to $98 \%$. Fig. 22 shows simulated measurements from an asteroid flyby with a cratered surface, and with detection probability of $85 \%$. Here $R$ is the measured range for each detected pulse, and $R_{0}$ is the detected at the first pulse. The shape of the surface can still be easily seen with $85 \%$ detection probability despite some missing points due to insufficient amplitude. The overall width of the 


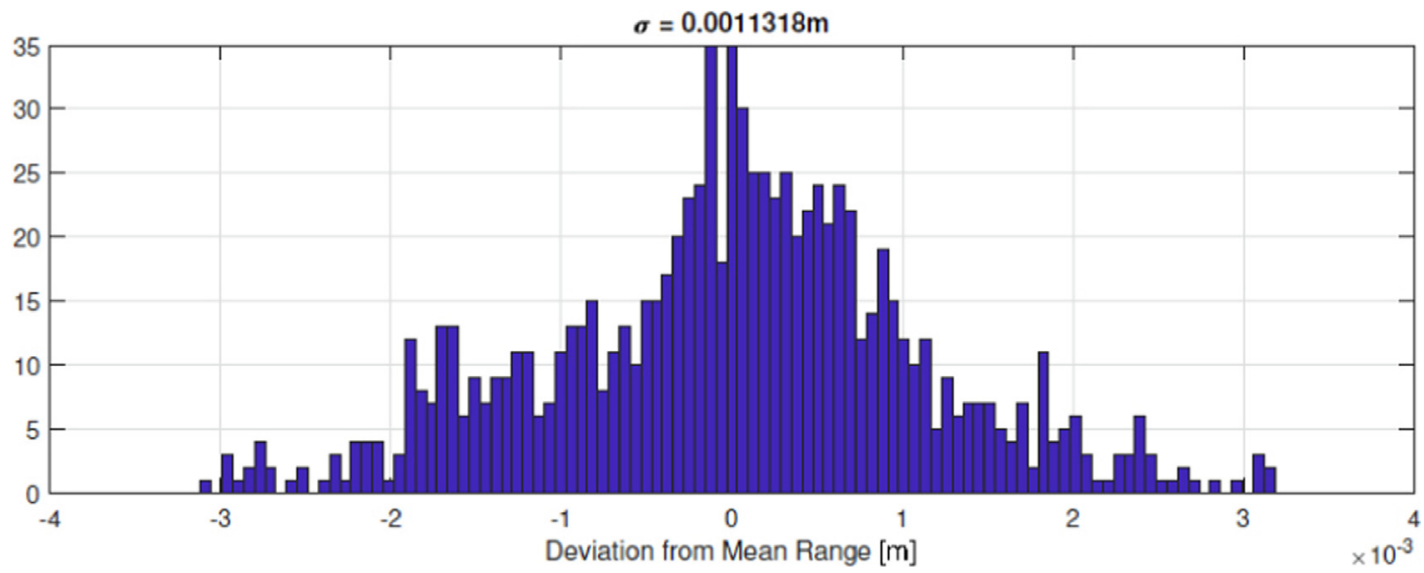

Fig. 20. Distribution of simulated range measurements for static-target ISR measurements.

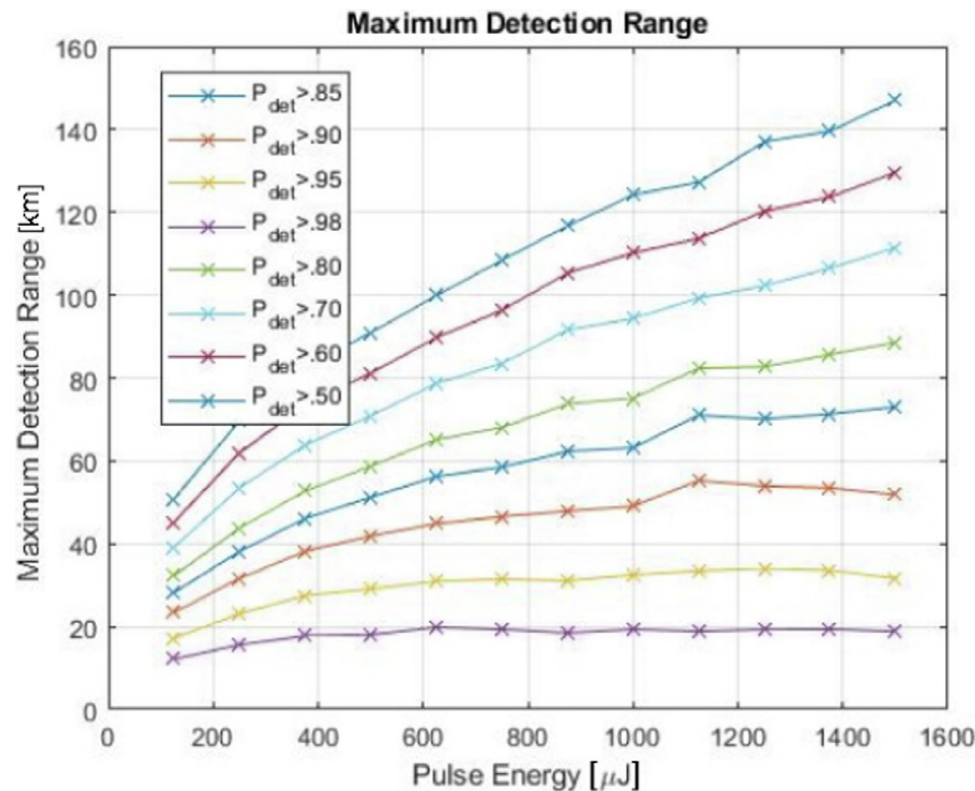

Fig. 21. Maximum reliable operational range for LIDARs of various pulse energies and acceptable individual pulse detection probabilities.

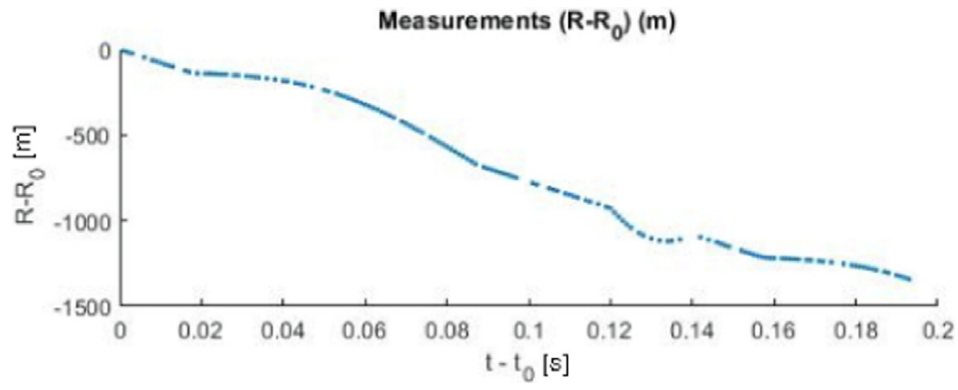

Fig. 22. Example of what a series of LIDAR measurements may look like for a cratered or bumpy surface. $R_{0}$ is the initial detection range. Craters in this case were generated by the addition of time-dependent sine terms to a flat-target result for the purpose of illustration.

object along the beam track (by number of pulses) can also be easily obtained from this curve. These are the only parameters that are required other than the absolute range to the asteroid.
In agreement with the preliminary design requirements of the NEACORE mission, in this paper the LIDAR is assumed to be $1 \mathrm{~kg}$ in mass and $1.5 \mathrm{U}$ in volume, where $\mathrm{U}$ refers to a standard CubeSat unit of 
$10 \mathrm{~cm} \times 10 \mathrm{~cm} \times 10 \mathrm{~cm}$. It can be seen in Fig. 21 that for low ranges in the up $20 \mathrm{~km}$ region, low pulse energies of $125 \mu \mathrm{J}$ are sufficient, even for a $98 \%$ pulse detection probability. This means that in reality the LIDAR may be able to use less volume and mass resources as it was initially roughly sized assuming a $1 \mathrm{~mJ}$ pulse energy. As mentioned, the direct ranging of the asteroid is redundant if both spacecraft survive the journey to the asteroid in formation, so if the LIDAR is sized only for ISR measurements it could use a significantly smaller collector and require mush lower pulse energy.

\subsubsection{Asteroid mass estimation}

The approach to determine the mass of an asteroid by dual flyby, requires the monitoring of the state of the two-spacecraft system (including spacecraft separation) along the trajectory before, during and after the flyby. The measured separation is then compared with the predictions coming from the propagation of a dynamic model with a variable asteroid mass to determine what mass gives the best fit of observation to prediction.

In this paper we have not simulated the whole guidance, navigation and control (GNC) sequence from close approach to post flyby conditions. Instead we introduced some simplifying assumptions that allowed us to quickly test the sensitivity of our approach against uncertainty in state of the spacecraft, mass of the asteroid and measurements.

The dynamics are described in an inertial heliocentric Cartesian coordinate system (see Fig. 23), where the $\mathrm{x}$ and $y$ axes are in the ecliptic plane. The orientation of the $\mathrm{x}$ and $\mathrm{y}$ axes in each simulation is chosen such that the node at which the spacecraft flies-by an asteroid is on the $x$ axis (see Fig. 2). Time is defined such that $-T_{c 1}$ is the start of the ISR measurements, and all trajectories are propagated from $-T_{c 1}$ to $+T_{c 2}$ (see in Fig. 2). The state vector of the two spacecraft is defined as follows:

$\mathbf{X}(t)=\left[\begin{array}{c}\mathbf{x}_{s c 1}(t) \\ \dot{\mathbf{x}}_{s c 1}(t) \\ \mathbf{x}_{s c 2}(t) \\ \dot{\mathbf{x}}_{s c 2}(t)\end{array}\right]$ where $\mathbf{x}_{s c 1}$ and $\dot{\mathbf{x}}_{s c 1}$ are respectively the position and velocity vectors of spacecraft 1 relative to the Sun, and similar notation is used for spacecraft 2 with subscript sc2.

The dynamics is governed by the following system of equations that accounts for both the effect of the Sun and the asteroid:

$$
\dot{\mathbf{X}}(t)=\left[\begin{array}{c}
\dot{\mathbf{x}}_{s c 1}(t) \\
-\mu_{s u n} \frac{\mathbf{x}_{s c 1}(t)}{\left\|\mathbf{x}_{s c 1}(t)\right\|^{3}}-\mu_{a s t} \frac{\left(\mathbf{x}_{s c 1}(t)-\mathbf{x}_{a s t}(t)\right)}{\left\|\mathbf{x}_{s c 1}(t)-\mathbf{x}_{a s t}(t)\right\|^{3}} \\
\dot{\mathbf{x}}_{s c 2}(t) \\
-\mu_{s u n} \frac{\mathbf{x}_{s c 2}(t)}{\left\|\mathbf{x}_{s c 2}(t)\right\|^{3}}-\mu_{a s t} \frac{\left(\mathbf{x}_{s c 2}(t)-\mathbf{x}_{a s t}(t)\right)}{\left\|\mathbf{x}_{s c 2}(t)-\mathbf{x}_{a s t}(t)\right\|^{3}}
\end{array}\right]
$$

where $\mu_{\text {sun }}=G m_{\text {sun }}$ and $\mu_{\text {ast }}=G m_{A}$ are the gravitational parameters of the Sun and the asteroid, $m_{A}$ is the mass of the asteroid and $G$ the universal gravity constant. The dynamics is then propagated using the MATLAB function ode45.

The initial conditions were defined such that the desired $1 \mathrm{~km}$ altitude close flyby occurs for each asteroid in the sequence. The procedure that follows was set up to guarantee the required close encounter and make the testing of the measurement phase repeatable within the limits of the simulated measurement error. In a more precise and realistic scenario one would need to simulate the whole GNC chain on approach to guarantee the proper targeting of the flyby conditions. However, we defer this more complex simulation to a future work and we limit ourselves only to the testing of the measurement capabilities of the two-way LIDAR system.

In line with this simulation strategy, we introduced two further simplifications. First, for all dual flyby simulations, the Keplerian elements of the asteroids in the reference tour were defined so that the asteroid is at the nominal position at the node at time $t=T_{0}$. This simplification removes the uncertainty on the exact trajectory of the asteroid and is required as we then propagate backwards to get initial conditions. However, as will be explained in the next section, we will re-introduce the asteroid position uncertainty by applying it to the initial conditions before forward repropagation.

The second simplifying assumption is that at $t=T_{0}$ both spacecraft are along the $x$ axis in the Ecliptic plane at the

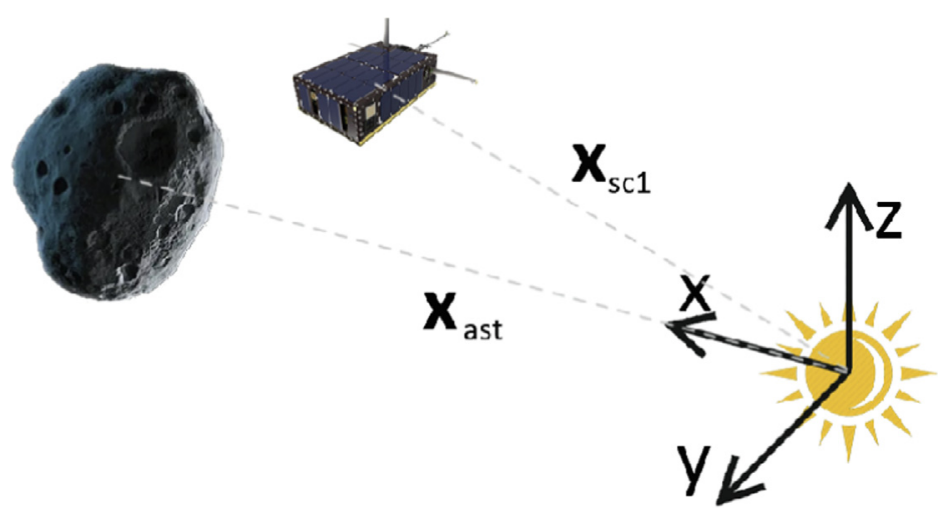

Fig. 23. Illustration of the coordinate system used. Coordinates are always Cartesian with the Sun at the origin. Flybys always occur at $y=0$. 
desired distance of $1 \mathrm{~km}$ from the asteroid. Thus their y coordinate is zero and the velocity vectors are in the Ecliptic plane and perpendicular to the $x$ axis. In reality, since the reference trajectory is slightly elliptical and the point of closest approach comes from a navigation and control sequence, the true state at $t=T_{0}$ will depart from this ideal situation. The magnitudes of the relative velocities at flyby in simulations differs slightly in some cases from those in the reference trajectory due to this simplification. Relative velocity magnitudes for simulations can be seen in the subfigure titles in Fig. 26. We will study the sensitivity to the actual point of closest approach by adding an uncertainty to the initial conditions as mentioned previously and explained in the next section.

Once the state vectors at $t=T_{0}$ were defined we propagated the trajectory of the spacecraft and the asteroid backward from $T_{0}$ to $-T_{c 1}$. The resulting state at time $-T_{c 1}$ was then taken as the nominal set of initial conditions. The nominal initial conditions are then propagated forward from $-T_{c 1}$ to $+T_{c 2}$, a period of 6 days (Fig. 2).

At each timestep in the state propagation the LIDAR measurements were simulated by reading the magnitude $R_{s c}(t)=\left\|\mathbf{x}_{s c 1}(t)-\mathbf{x}_{s c 2}(t)\right\|$ of the separation between the two spacecraft. Then, normally distributed noise terms with standard deviation $1.13 \mathrm{~mm}$ (derived in Section 5.2) were added to these exact truth values. Let us call the measured range $\widehat{R}$. We verified that the pointing errors do not impact the range measurement, as the beam divergence can easily be made larger than the pointing error to guarantee the other spacecraft is inside the beam radius. Furthermore we checked that, assuming a pointing accuracy of $1 \mathrm{mrad}, 3$ sigma, due to the attitude control system, the range error due to the pointing error is less than $1.13 \mathrm{~mm}$ on average. Thus only a one-dimensional measurement of range from the LIDAR was used. Measurements were generated after the propagation of the states and were not assumed to influence the propagation as the engines will be turned off and no more correction maneuvers are made in the measurement phase.
The effect of the mass of the asteroid on the trajectory of the spacecraft can be seen in Fig. 24 where $\widehat{R}$ is plotted for the same flyby and two different masses. In this case we assumed that the free, uncontrolled motion starts 1 day before the $T_{0}$ and ends 5 days after $T_{0}$. In order to determine the exact mass of an asteroid, one needs to find the value $m_{A}$ in Eqs. (14) so that the simulated measurements $R$ match the actual measurements $\widehat{R}$ as closely as possible. Since measurements are affected by an error one needs to minimise the cost function:

$\epsilon(z)=\sum_{i=1}^{N_{\text {samples }}}\left(\widehat{R}_{i}\left(m_{A}\right)-\bar{R}_{i}(z)\right)^{2}$

with:

$\bar{R}_{i}(z)=R_{s c}\left(t_{i}, z\right)+N_{s}\left(\sigma_{I S R}\right)$

and:

$\widehat{R}_{i}\left(m_{A}\right)=R_{\text {true }}\left(t_{i}, m_{A}\right)+N_{t}\left(\sigma_{\text {ISR }}\right)$

where $\bar{R}_{i}(z)$ is the simulated ISR measurement for test mass $z, \hat{R}_{i}\left(m_{A}\right)$ is the true measurement due to mass $m_{A}, R_{s c}\left(t_{i}, z\right)$ the noise-free intersatellite range at measurement time $t_{i}$ for asteroid mass $z$, and $N_{t}\left(\sigma_{I S R}\right)$ are normally distributed uncorrelated random noise terms with $\sigma_{I S R}=1.13 \mathrm{~mm}$ as derived earlier, newly generated for every individual simulated LIDAR range measurement. (Fig. 20). In a real scenario, the true measurements $\widehat{R}_{i}\left(m_{A}\right)$ are the real range data that would be collected from the flyby (and hence is a function of the true mass), and $\bar{R}_{i}(z)$ are the simulated measurements that would come from the on-board propagation of dynamics with an asteroid of mass $z$. Since we assume that the sensor is fully calibrated, we assumed that the simulated measurements can be derived from the propagated range $R_{s c}(t, z)$ augmented by the same noise the true measurements are affect by.

In this work we used a simple grid search on the values of $z$ to get a first guess and then a second, finer grid search to fine tune the mass estimate. In the simulations, measure-

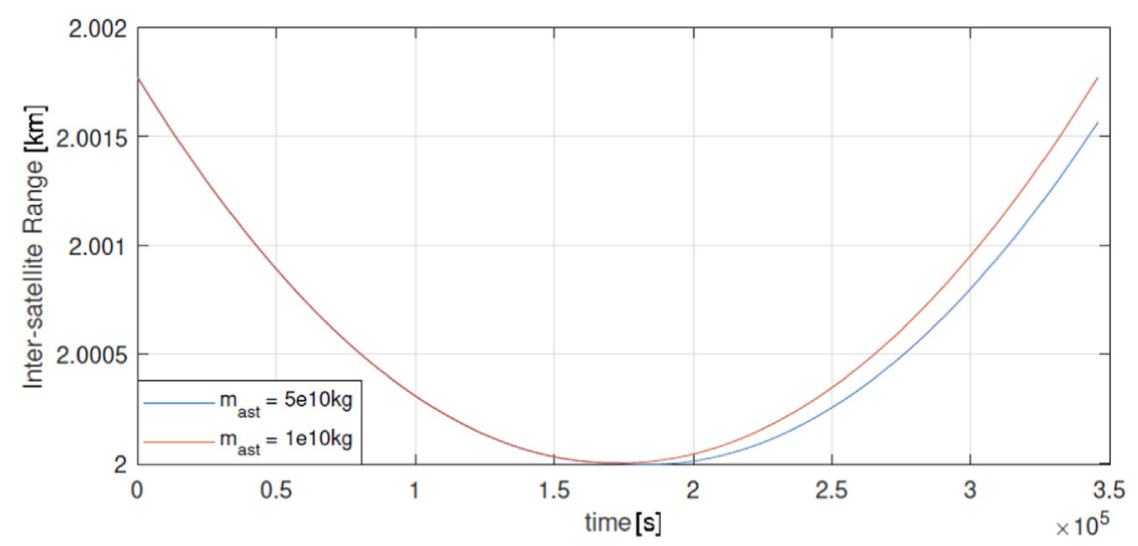

Fig. 24. Change in ISR truth curve for different asteroid masses when propagating the same initial condition. Timescale goes from flyby-2 days to flyby +2 days. 
ment samples were taken at each instant of time returned by ode 45 . Because the solver chooses different sample times for each propagation depending on the trajectory and the mass of the asteroid, any mismatches in sample times in different simulations are corrected by interpolating the truth curve for the sample times in the measurement set, so all simulations $R_{i}(z)$ have their measurements compared with the correct point in the truth curve. Figs. 25 show the residuals $\epsilon$ for two different examples. It can be seen that there is a clear reduction in $\epsilon$ around the true mass. The value taken for the true mass is simply that corresponding to the smallest overall value of $\epsilon$. As the true mass reduces, the width of this dip increases, thus increasing the uncertainty of the measurement. For all tested asteroids with a mass over $10^{9} \mathrm{~kg}$, the point of the minimum is clearly localised. For lower masses the identification of an exact value is more problematic and the residuals are more significant.

In all cases it can be seen that $\epsilon$ maintains an approximately constant value below masses of approximately $3 \times 10^{8} \mathrm{~kg}$ before starting to decrease to a minimum. This indicates that the effect of the asteroid gravity below this mass becomes immeasurably small over the simulated timescale of $T_{c 2}-T_{c 1}=6$ days for this method. However, at all tested masses above this value, very good measurement accuracy is obtained, typically on the order of $1 \%$ relative error or less, and $20 \%$ relative error in the case of the $5.69 \times 10^{8} \mathrm{~kg}$ asteroid, which is approaching the lower limit of detectability.
The limits of the technique to estimate the mass of the asteroid were further assessed by determining the relative error in the estimation the mass for each asteroid in the reference tour. The expectation is to see a sensitivity to the flyby velocity. For each flyby, however, we did not use the actual expected mass of the asteroid but we varied the mass from $10^{8}$ to $10^{12} \mathrm{~kg}$. The results of this analysis can be seen in Fig. 26. Here the individual data points represent different runs of the simulation and the solid line connects the mean relative error of each mass. It can be seen that for the 3 fastest flybys (subfigures A, B and D) if the mass is $10^{8} \mathrm{~kg}$, an estimation error on the order of $100 \%$ is expected. For the slowest flyby (subfigure C), on the order of $10 \%$ relative error is expected for the same mass. These expected errors decrease with asteroid mass as expected, with asteroids over $10^{10} \mathrm{~kg}$ having an expected error of order $1 \%$ in all cases.

\subsubsection{Sensitivity to initial conditions and asteroid mass}

Up until this point, the nominal initial conditions were used to assess the accuracy of the mass estimation.

However, in a real mission scenario one has to account for the uncertainty in the initial conditions when the ISR phase starts, as this will affect the actual distance from the asteroid. Likewise, given that the mass of the asteroid is uncertain, the dynamics can bring the spacecraft closer to the asteroid or leave them farther away than expected.
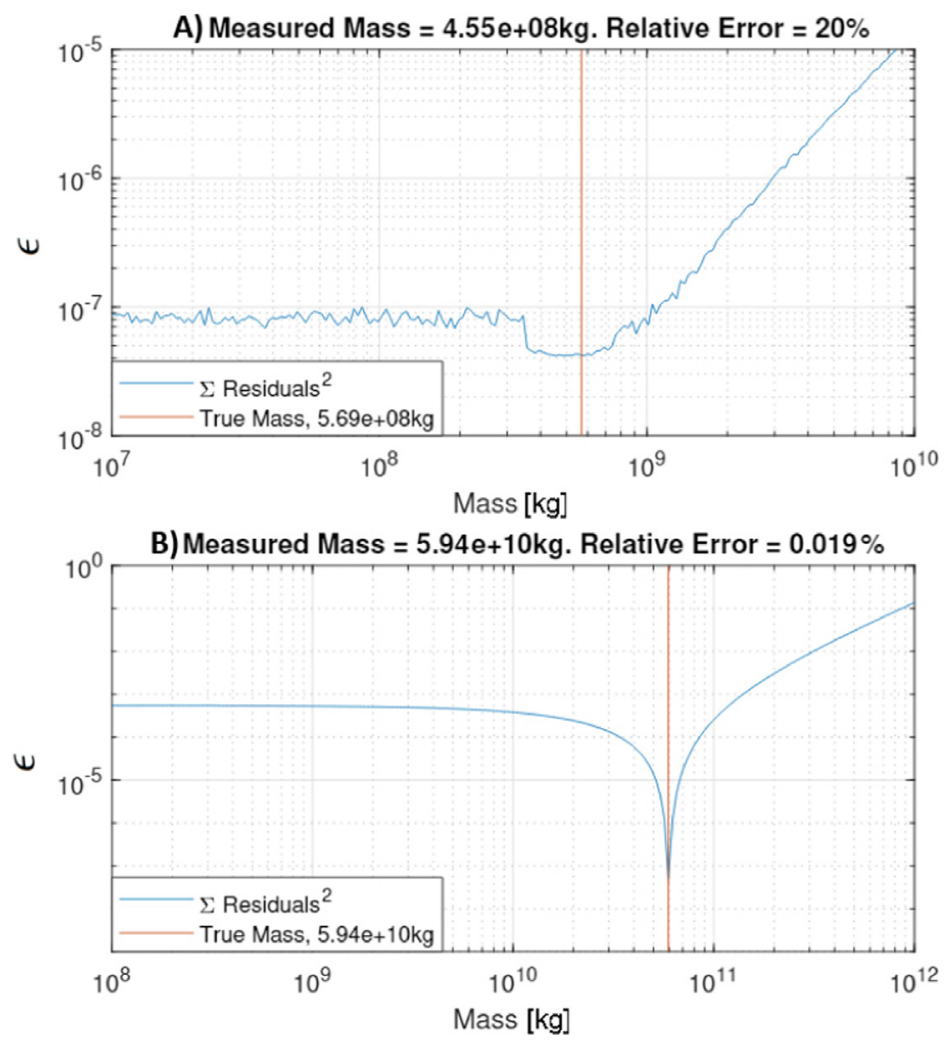

Fig. 25. Residuals for $5.69 \times 10^{8} \mathrm{~kg}(\mathrm{~A})$ and $5.94 \times 10^{10} \mathrm{~kg}(\mathrm{~B})$ asteroids showing the evolution of the residual distribution with mass. 

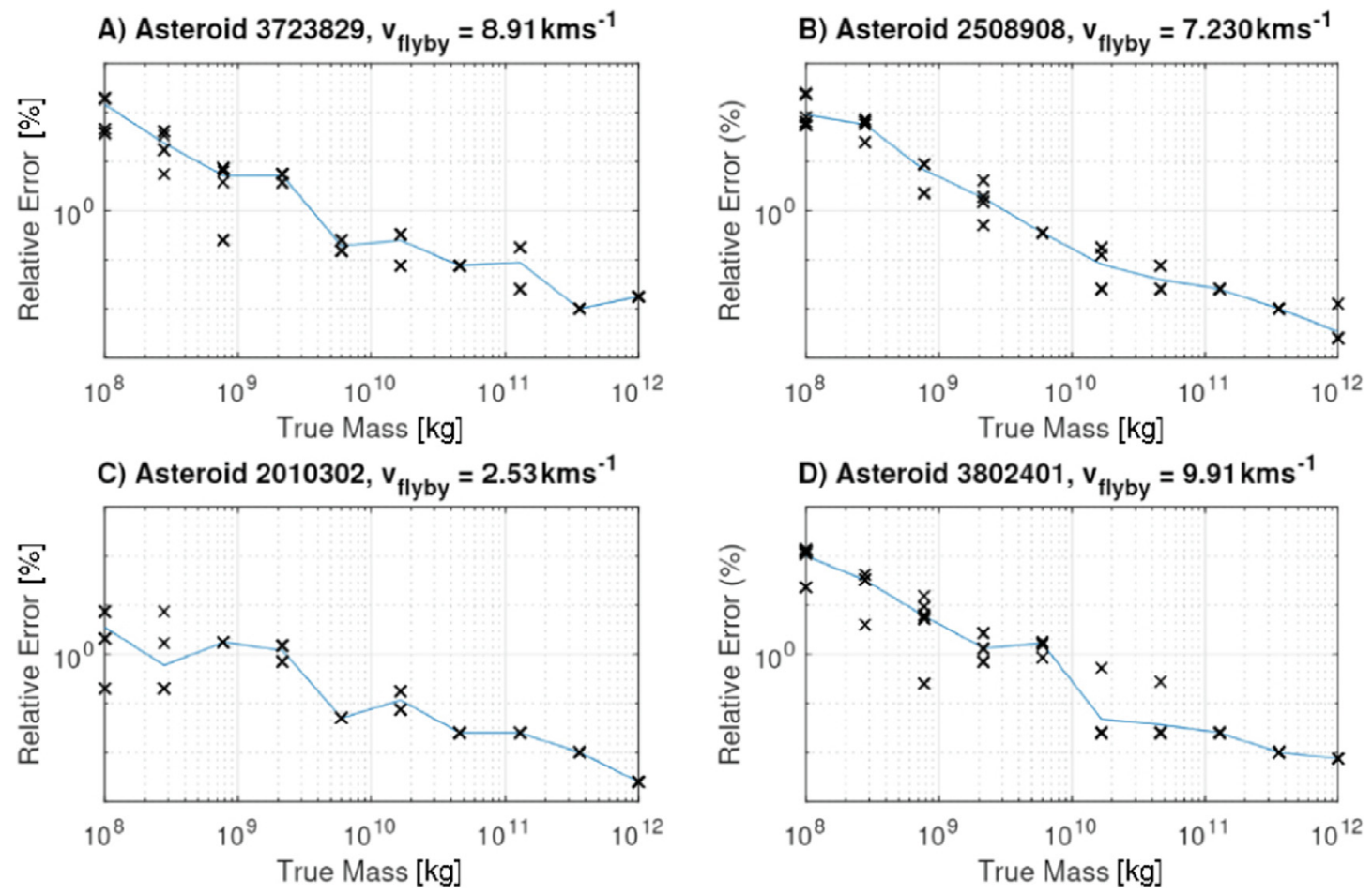

Fig. 26. Relative error on mass estimation for each asteroid in the reference sequence, for various values of their true masses with a $1 \mathrm{~km}$ flyby altitude. Crosses represent different runs and the solid line connects the mean error at each mass.

Thus, a Monte-Carlo simulation of 1000 flybys each for 13 different asteroid masses between $10^{8}-10^{20} \mathrm{~kg}$ was performed, with different random variations added to both the initial position and velocity components of the spacecraft. Note that we assume that the relative position and velocity of the spacecraft can be known and controlled very accurately. Hence, the error in initial conditions is the same for both spacecraft and comes from the re-targeting phase.

Fig. 27 shows the distribution of true flyby distances for each asteroid mass, when noise terms are added to the initial condition with standard deviations on position and velocity components of $\sigma=5 \mathrm{~km}$ and $\sigma=10 \mathrm{~cm} / \mathrm{s}$ respectively as order-of-magnitude estimates of the possible uncertainty of the state elements relative to the asteroid from the guidance, navigation and control subsystem. These estimates were obtained by looking at past missions and concepts for deep space navigation relative to asteroids (Ozaki et al., 2016) (Accomazzo et al., 2010) (Hashimoto et al., 2010) (Cui and Zhu, 2014). Fig. 27 is welldescribed by a Rayleigh distribution with a mode value of $9.8 \mathrm{~km}$ closest approach. When each mass is plotted on a separate histogram it can be seen that the mass does

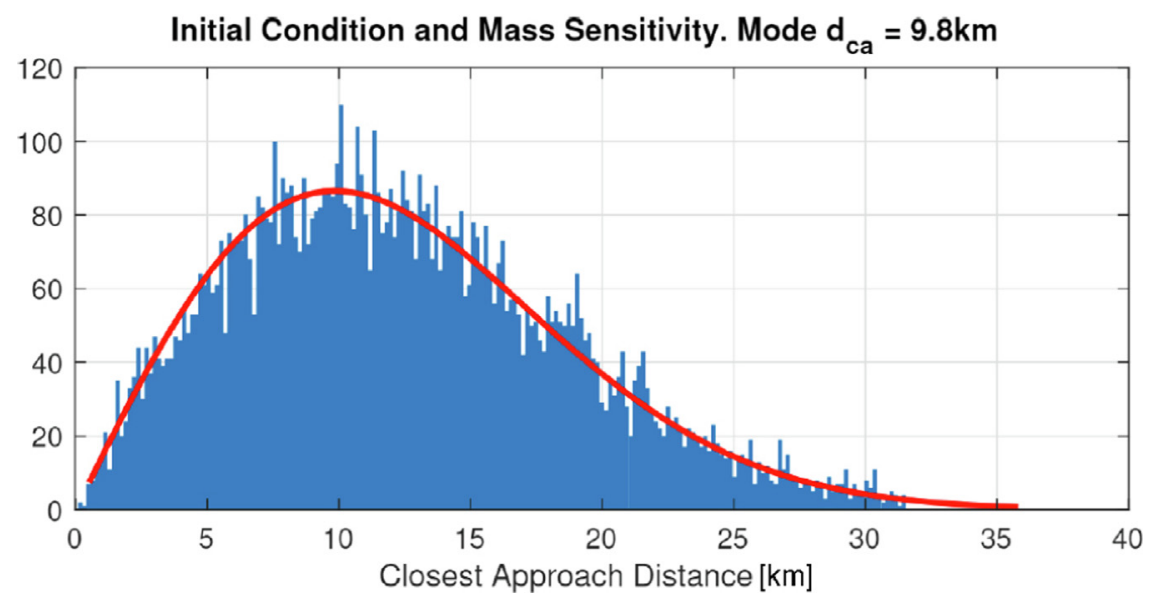

Fig. 27. Sensitivity to initial orbital conditions with 1000 iterations for 9 different asteroid masses from $10^{8}$ to $10^{16} \mathrm{~kg}$. When plotting masses independently it can be seen that the mass does not cause any significant change in the mode of the distribution. The most likely actual flyby distance is $9.8 \mathrm{~km}$. 


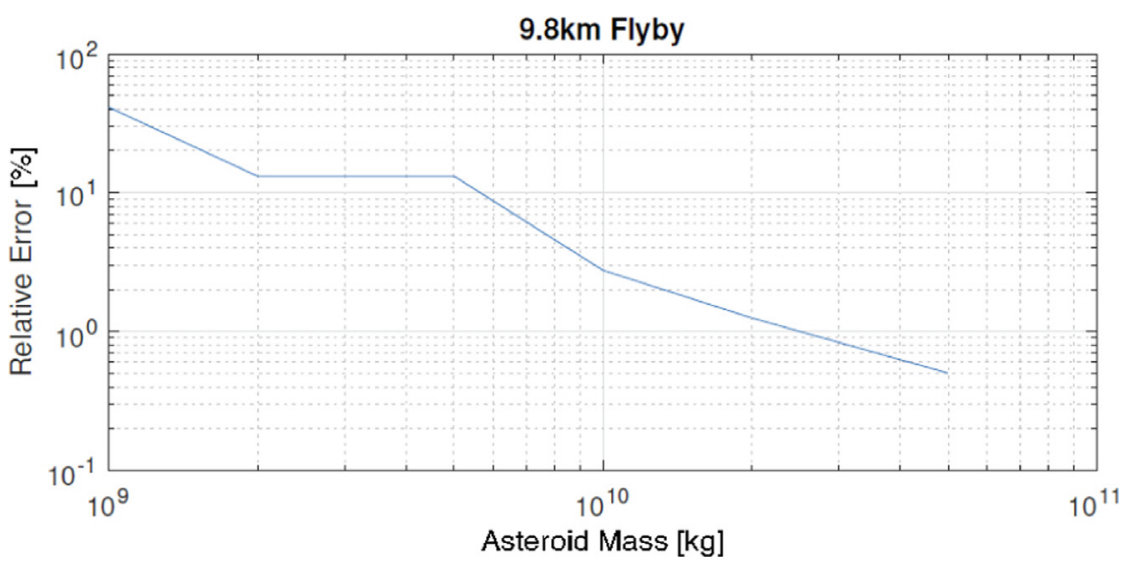

Fig. 28. Mean relative error for different masses when flying by at the expected distance of $9.8 \mathrm{~km}$.

not actually cause any significant change in the flyby distance. These separated histograms can be found in the appendix (Fig. A.36).

From the sensitivity analysis in Fig. 27, it can be seen that when targeting a $1 \mathrm{~km}$ flyby, the likely true flyby distance will be around $9.8 \mathrm{~km}$ under this level of uncertainty. This will result in weaker gravitational interaction than a close flyby, and so $9.8 \mathrm{~km}$ flybys were also tested and compared with $1 \mathrm{~km}$ flybys for the first asteroid in the reference tour to determine the mass limit in the $9.8 \mathrm{~km}$ case.

The relative error on the mass measurement for different masses when flying by at $9.8 \mathrm{~km}$ is shown in Fig. 28. When looking at the residual curve for each mass, it was found that below about $10^{9} \mathrm{~kg}$, no discernible dip in the curve was present, and the algorithm had just chosen the minimum value in a noisy straight line, indicating the trajectory had not been sufficiently deflected to be measured in the simulated timescale. Thus for the more realistic flyby distance of $9.8 \mathrm{~km}$, the lower limit of mass that could be detected in this way is around $10^{9} \mathrm{~kg}$, about an order of magnitude larger than the limit when a $1 \mathrm{~km}$ flyby can be successfully achieved. In this assessment we included the error coming from the unknown relative drift of the two spacecraft due to the fact that they are not on the expected trajectory.

This indicates that when pushing the limits of this technique for smaller and lighter asteroids, it is imperative to be able to ensure a closer flyby occurs, such that the deflection is stronger. A remediation solution is to delay the cut-off point $-T_{c 1}$ so that the spacecraft can manoeuvre and get as close as possible to the asteroid. This strategy is similar to current targeting strategies for kinetic impactors.

\section{Discussion}

From the analyses in this paper we can derive some requirements for the mission concept we propose. Firstly, it was found that the trajectory deviation is strongly dependent on asteroid mass, as expected. When using the leastsquares method for fitting a model to the observations, as the mass approached the lower limit of measurability, the error increased. For faster flybys this was more significant an effect. Thus a requirement can be derived that the mission cannot target asteroids with an expected mass less than $10^{8}-10^{10} \mathrm{~kg}$, however the exact value of this minimum is highly dependent on the expected flyby velocity and the required level of accuracy in the mass estimation. This is up to mission planners to decide.

Another requirement was found that access to highprecision equipment for satellite orbit determination must be included in mission planning, in order to ensure a sufficiently close flyby is achieved to cause detectable trajectory deflection. The flyby distance of $9.8 \mathrm{~km}$ used in this analysis was derived assuming $1 \sigma$ uncertainties on the satellite position and velocity relative to the asteroid of $5 \mathrm{~km}$ and $10 \mathrm{~cm} / \mathrm{s}$. Although Earth based antennas can track the satellite's position to far better accuracy than this, the limiting factor is navigation relative to the asteroid. The predicted expected flyby distance in this mission concept of $9.8 \mathrm{~km}$ is very close to that achieved in the Rosetta flyby of asteroid 2867 Steins, which was able to fly by within $6.6 \mathrm{~km}$ of its target point (Accomazzo et al., 2010) (note that our target point is much closer to the asteroid) while on a similar approach velocity of $8.6 \mathrm{~km} / \mathrm{s}$ and performing its last maneuver $36 \mathrm{~h}$ before flyby. Rosetta also informed its correction maneuvers using the help of on-board optical observations, which is overall a very similar mission profile.

A third requirement is that high-precision LIDARs must be present on both satellites. The spacecraft separation measurement accuracy is a function of the laser pulsewidth and SPAD array time bin width. Lower values for both of these while maintaining the same pulse energy lead to more accurate ranging, and values of $t_{\text {pulse }}=100 \mathrm{ps}, t_{\text {bin }}=10 \mathrm{ps}$ were used in this paper which are very reasonable numbers to obtain, and by our analysis result in a $1 \sigma$ range error of $1.13 \mathrm{~mm}$. This is on par with existing hardware, such some systems produced by Jenoptik (chosen for use on the M-ARGO CubeSat), which have accuracy on the millimeter level. Although laser altimeters have been ubiquitous on Earth observation satellites and 
planetary and lunar missions in the past, they have not yet been widely used on nanospacecraft to take advantage of advances in miniaturised systems in recent years. The planned Asteroid Impact Mission (Wolters et al., 2011) uses NEAR heritage technology for its laser altimeter and is rather large and heavy, however is should be noted that AIM and instruments on other past missions such as the NEAR Laser Rangefinder, Mars Orbiter Laser Altimeter, Lunar Orbiter Laser Altimeter, Mercury Laser Altimeter all perform direct ranging by surface scattering rather than the two-way ranging approach proposed in this paper, vastly increasing the pulse energy requirement and hence size and mass of the laser system. Spacecraft pointing drift could also add some noise to the range measurements, and although this was not investigated in this paper, a method was suggested to maintain a good pointing angle if the imaging camera can be made sensitive to the LIDAR wavelength, or an optical wavelength is chosen for the LIDAR. It was found that by using the two-way ranging method one can vastly reduce the pulse energy requirement from the $\mathrm{mJ}$ to the $\mu \mathrm{J}$ level.

A frame summation algorithm such as that proposed in Lightholder et al. (2019) must be developed in order to allow long compound exposures to be used to acquire an image of the asteroid sufficiently early to allow TCMs to be performed. This would be the first time such a technique would have been used for navigation, however Earth-based and space-based telescopes already use this technique for astronomical observations to improve SNR. Other strategies to ensure the close flyby can be achieved include further ephemeris improvement from telescopes on Earth or in orbit prior to the launch of the mission, reducing the $3 \sigma$ ellipsoids and allowing optical acquisition to occur closer to the flyby. Also the target selection process could be restricted to only include asteroids with better known orbits, although this would likely remove many of the smaller asteroids from the list of possible targets.

The measurement period simulated here was from one day prior, to 5 days after flyby. It should be noted that if the measurement period is extended for longer after the flyby, it should be possible to measure mass more precisely (or to smaller values) as the trajectory deviation compounds over time.

The available tours found in Section 3 were chosen with a $\Delta V$ requirement of $<1 \mathrm{~km} / \mathrm{s}$ for the IO transfer. Further corrections were allowed to achieve the flybys, but these were not allowed to exceed $2 \mathrm{~km} / \mathrm{s}$, for a total $\Delta V$ requirement of the tour of $<3 \mathrm{~km} / \mathrm{s}$. If more $\Delta V$ could be achieved with a given spacecraft design, this would open up a wider number of possible targets, tours, departure dates, and number of asteroids per tour, although different $\Delta V$ budgets were not studied in detail here. It should also be noted that the set of IOs investigated in Section 3 was relatively limited, and finer spacing of the IO orbital elements could have led to more possible tours being identified.
We can see that the proposed dual flyby would allow one to gather essential information to study the future evolution of the targets. Besides updating and improving the ephemerides, each close flyby can be used to improve our knowledge of the physical quantities that define the Yarkovsky (Tardioli et al., 2017) and YORP effects (Bottke et al., 2006). These two effects are dictated by a number of physical parameters that are difficult to directly measure from Earth.

From a fast flyby we can obtain good measurements of the size of the object, its mass (as demonstrated in this paper), the surface optical properties, the obliquity and the Bond albedo. The last two measurements, although not considered in this paper, are possible and, together with the mass, would allow one to significantly improve the dynamic model of the asteroid.

\section{Conclusions}

In this work we have proposed a mission concept for the large-scale low-cost exploration of Near Earth Objects. The main contribution of this paper is to demonstrate that with the proposed mission concept we can visit multiple asteroids, within a given size range and time frame, and extend the range of scientific information that can be gathered from ground observations. We focused in particular on a new way to determine the mass of NEOs.

The mission concept consists of multiple flybys of NEOs with pairs of identical $12 \mathrm{U}$ cubesats, equipped with electric propulsion, a LIDAR and a camera, flying in formation. We have shown that a large number of possible multiasteroid tour trajectories can be generated to suit the proposed mission concept under the constraints of a total $\Delta v<3 \mathrm{~km} / \mathrm{s}$, a $v_{f l y b y}<10 \mathrm{~km} / \mathrm{s}$ and the requirement that at least one asteroid in a sequence is greater than $150 \mathrm{~m}$ in diameter. The generated tours in the time frame studied have up to 15 objects, however 3-5 is more typical, which could be taken as the expected number of asteroids per tour.

We have also shown that a camera suitable for a $12 \mathrm{U}$ CubeSat is capable of acquiring targets sufficiently far in advance to allow for orbit determination and likely lowthrust trajectory corrections to enable close flybys. An impulsive propulsion system may be required to fine tune the targeting of the required flyby conditions.

Finally, we have shown with a preliminary analysis that the proposed method for asteroid mass characterisation using LIDAR to measure the relative position of the two spacecraft is feasible for asteroid masses greater than approximately $3 \times 10^{8} \mathrm{~kg}$ at a flyby velocity of $8.931 \mathrm{~km} /$ $\mathrm{s}$ if a $1 \mathrm{~km}$ flyby altitude can be achieved, and approximately $1 \times 10^{9} \mathrm{~kg}$ for the same velocity if a further flyby of $9.8 \mathrm{~km}$ is the best that can be achieved. This flyby velocity is towards the upper limit of the range of asteroids included in the initial target selection phase and it was also shown that slower flybys will result in stronger and more 
detectable deflections at lower asteroid masses, changing the lower mass limit of the technique.

From the results in this paper, one can conclude that the proposed mission concept, and in particular its novel approach for measuring the mass of a NEO, can improve our knowledge of some key physical properties of a large number of NEOs. The next step for the analysis of this concept is a full simulation of the GNC chain along the actual tour trajectory. This will allow a more precise analysis of the lowest flyby altitude that can be achieved with a $12 \mathrm{U}$ CubeSat.

\section{Declaration of Competing Interest}

The authors declare that they have no known competing financial interests or personal relationships that could have appeared to influence the work reported in this paper.

\section{Acknowledgements}

Thanks to the University of Strathclyde and Fraunhofer CAP UK for providing funding for this work, as well as Pierre Bousquet (CNES), Franco Lissi (ESA) and Boris Segret (l'Observatoire de Paris) for providing assistance in the initial CDF study in which this mission was first developed.

\section{Appendix A. Additional figures}

Figs. A.29-A.36.
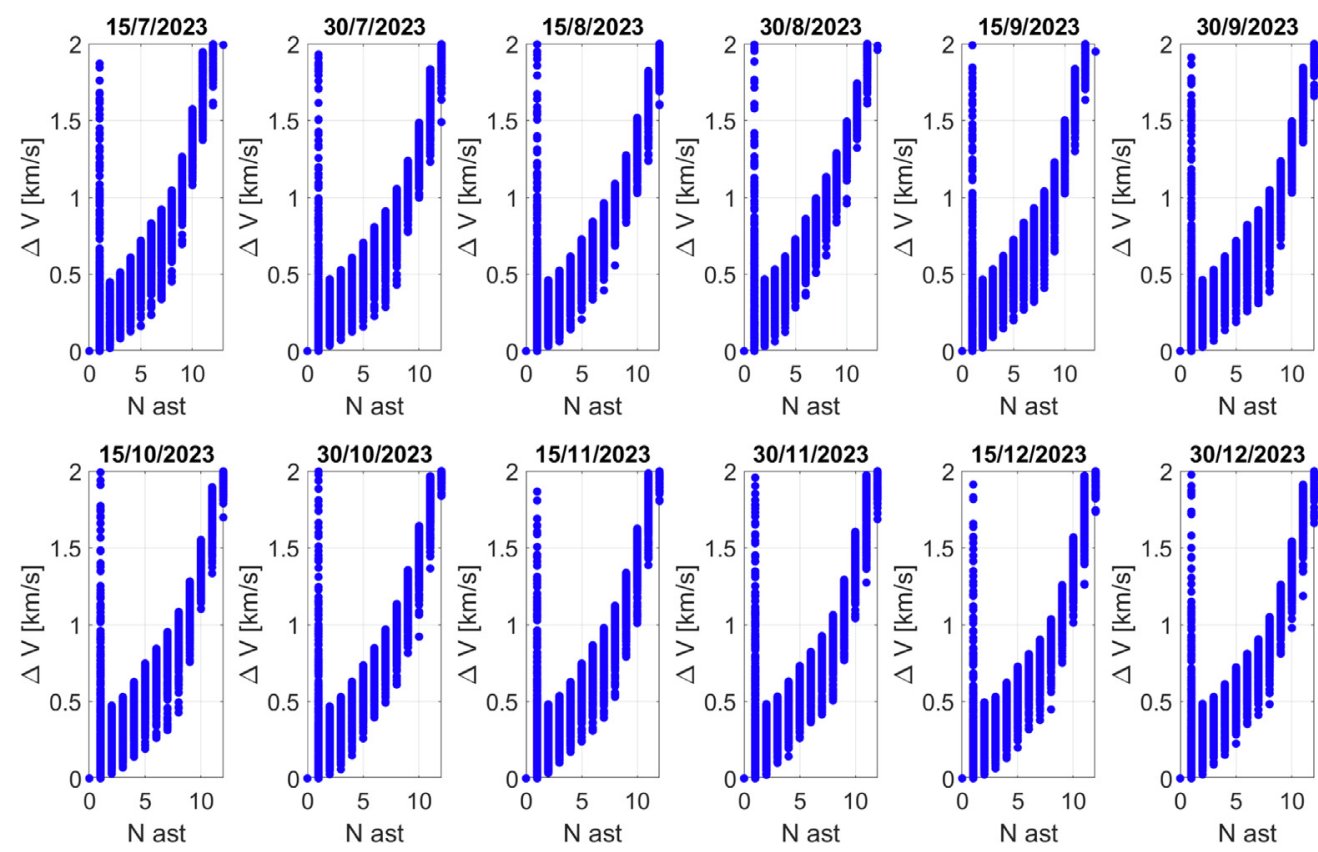

Fig. A.29. Solutions for orbit 1, from July to December 2023. 

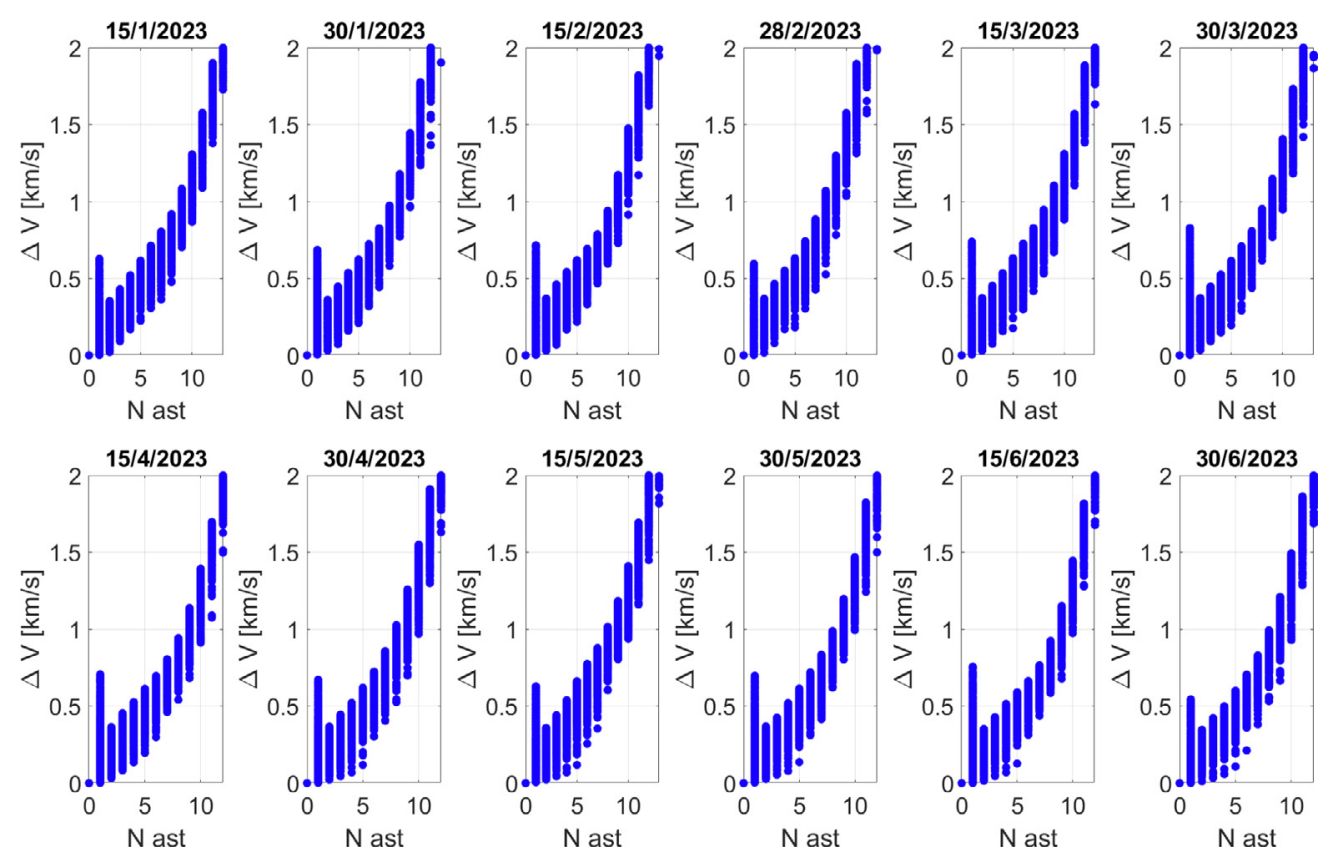

Fig. A.30. Solutions for orbit 2, from January to June 2023.
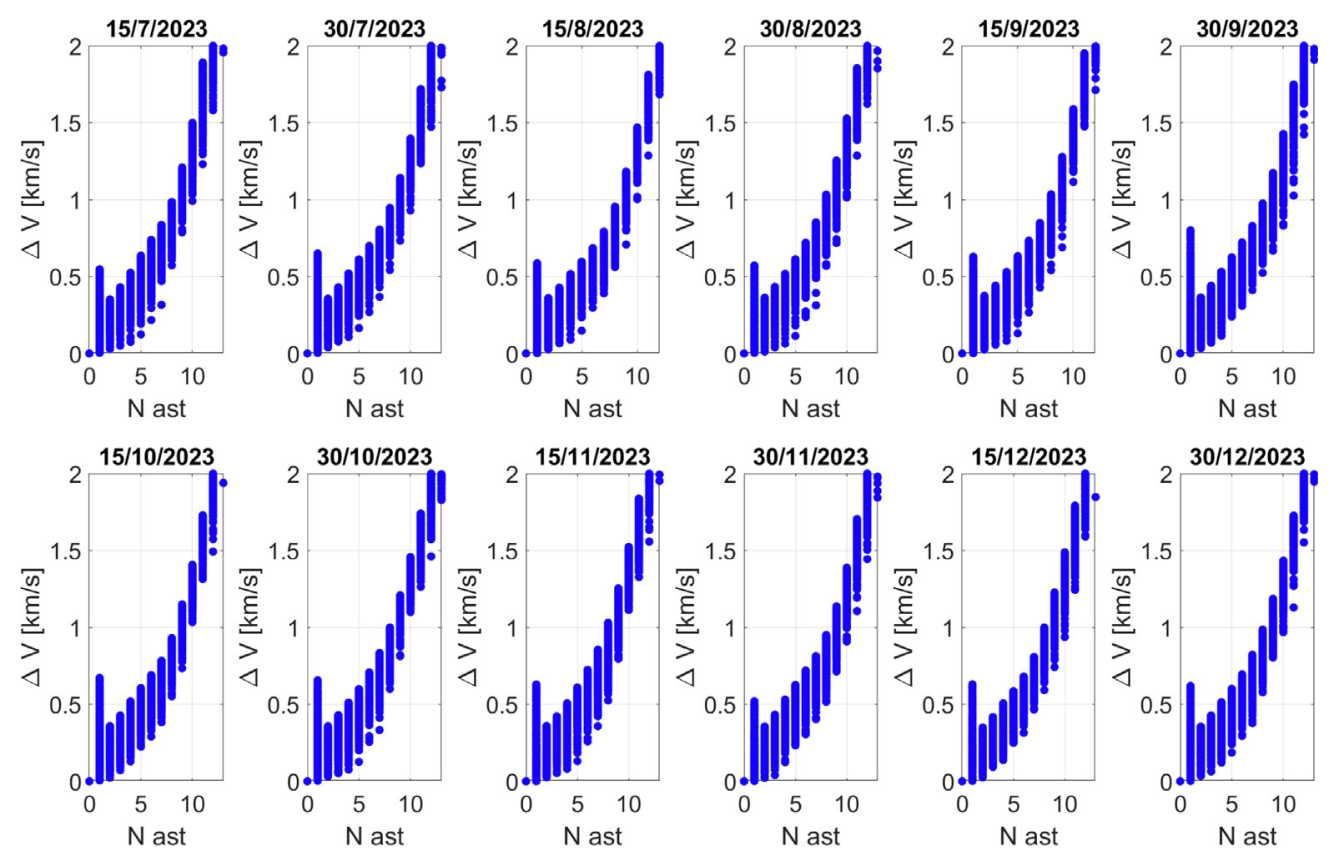

Fig. A.31. Solutions for orbit 2, from July to December 2023. 

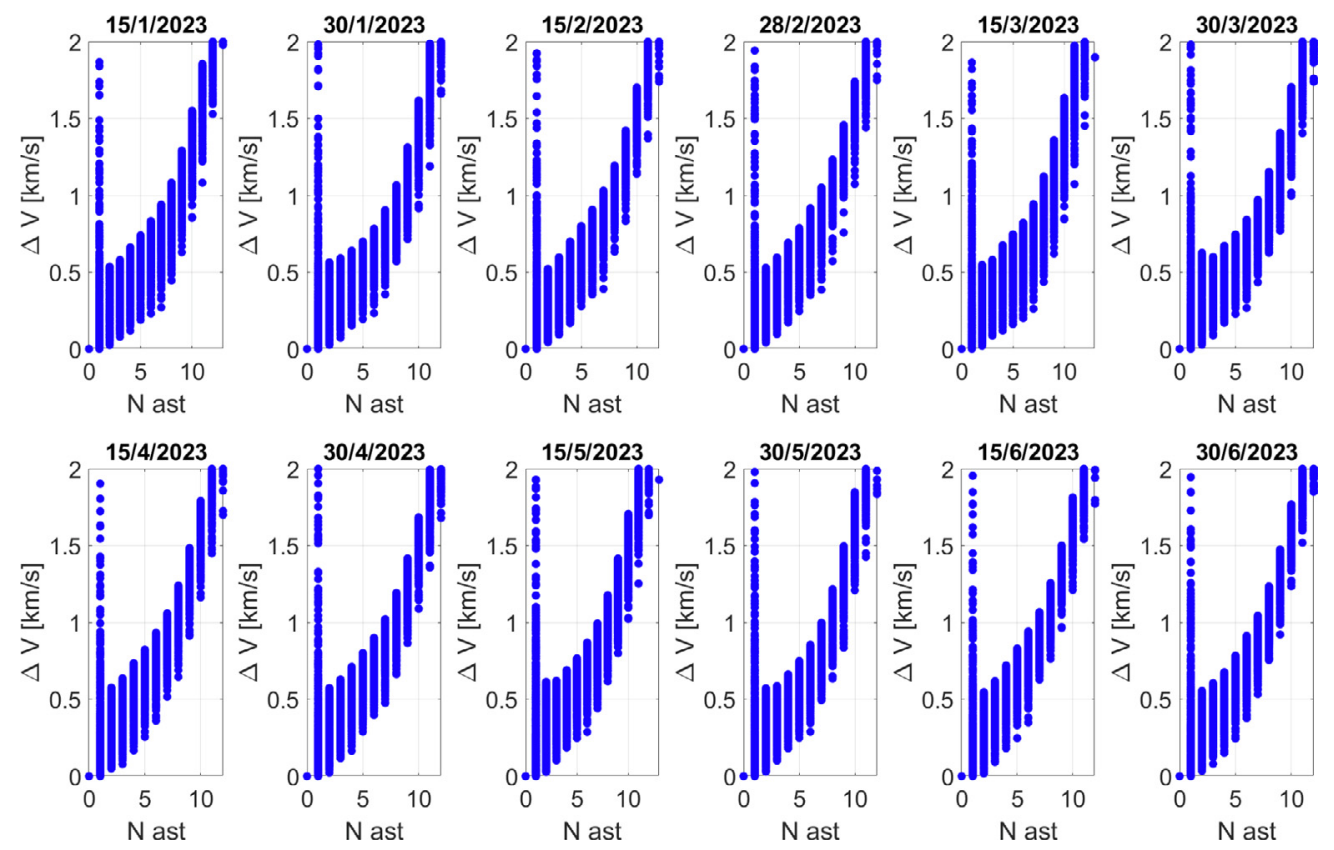

Fig. A.32. Solutions for orbit 3, from January to June 2023.
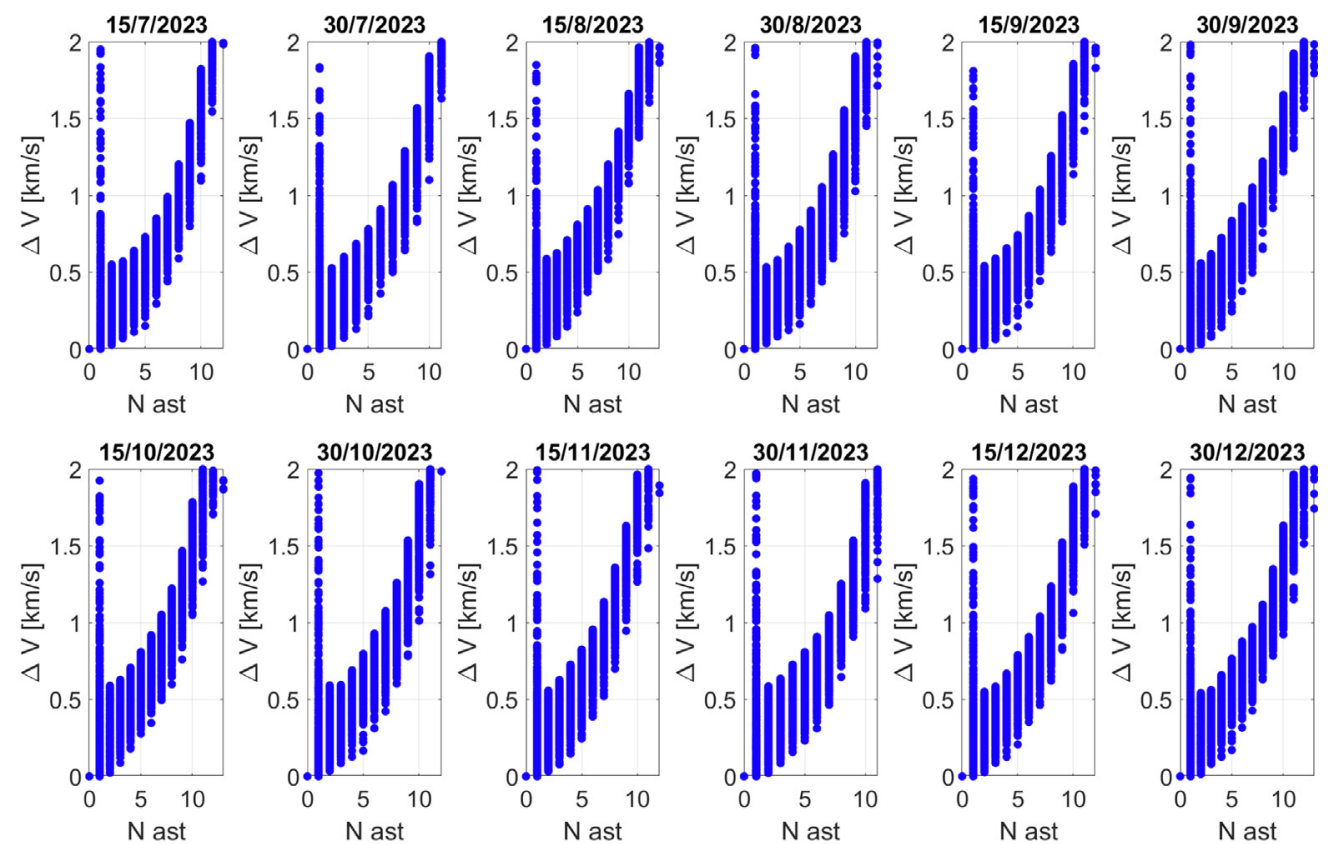

Fig. A.33. Solutions for orbit 3, from July to December 2023. 

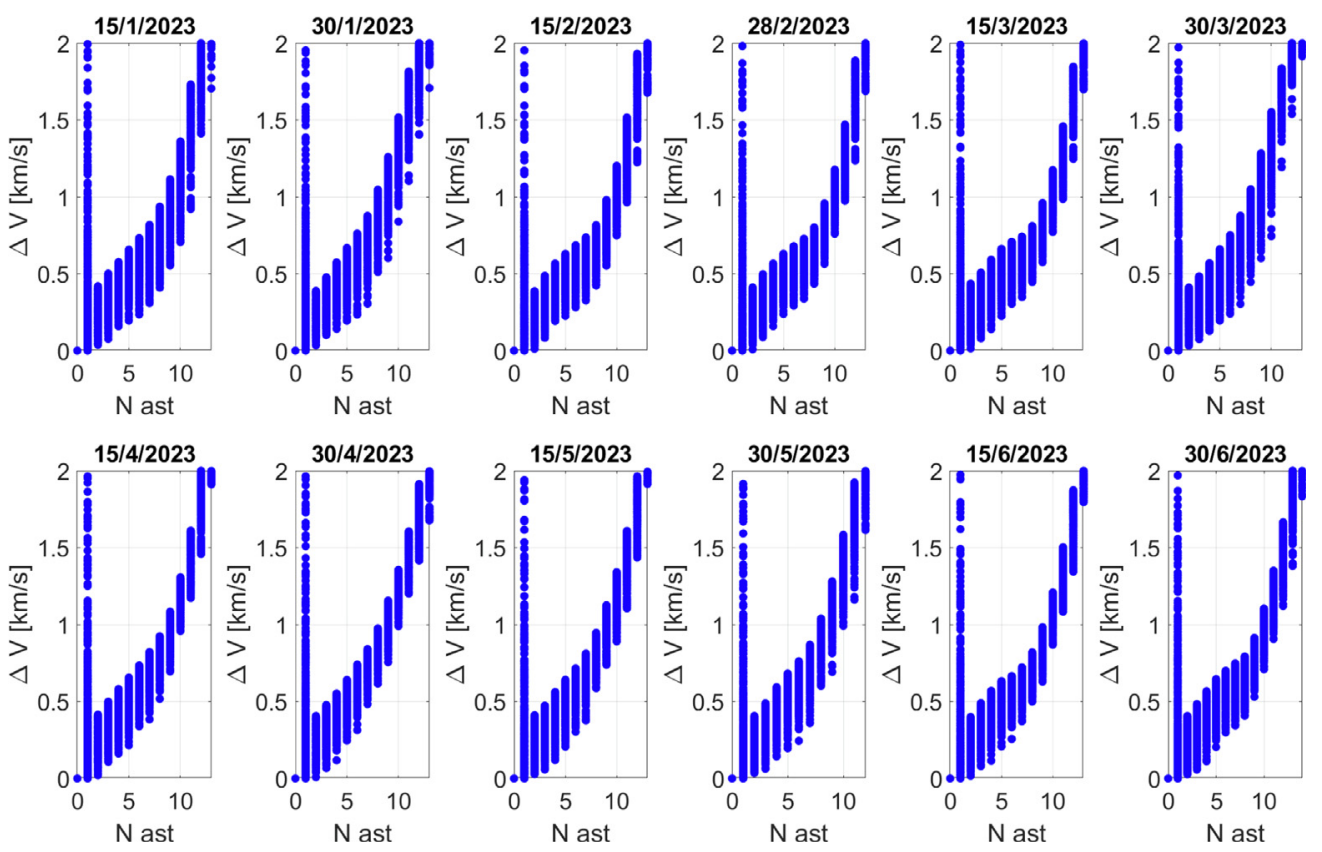

Fig. A.34. Solutions for orbit 4, from January to June 2023.
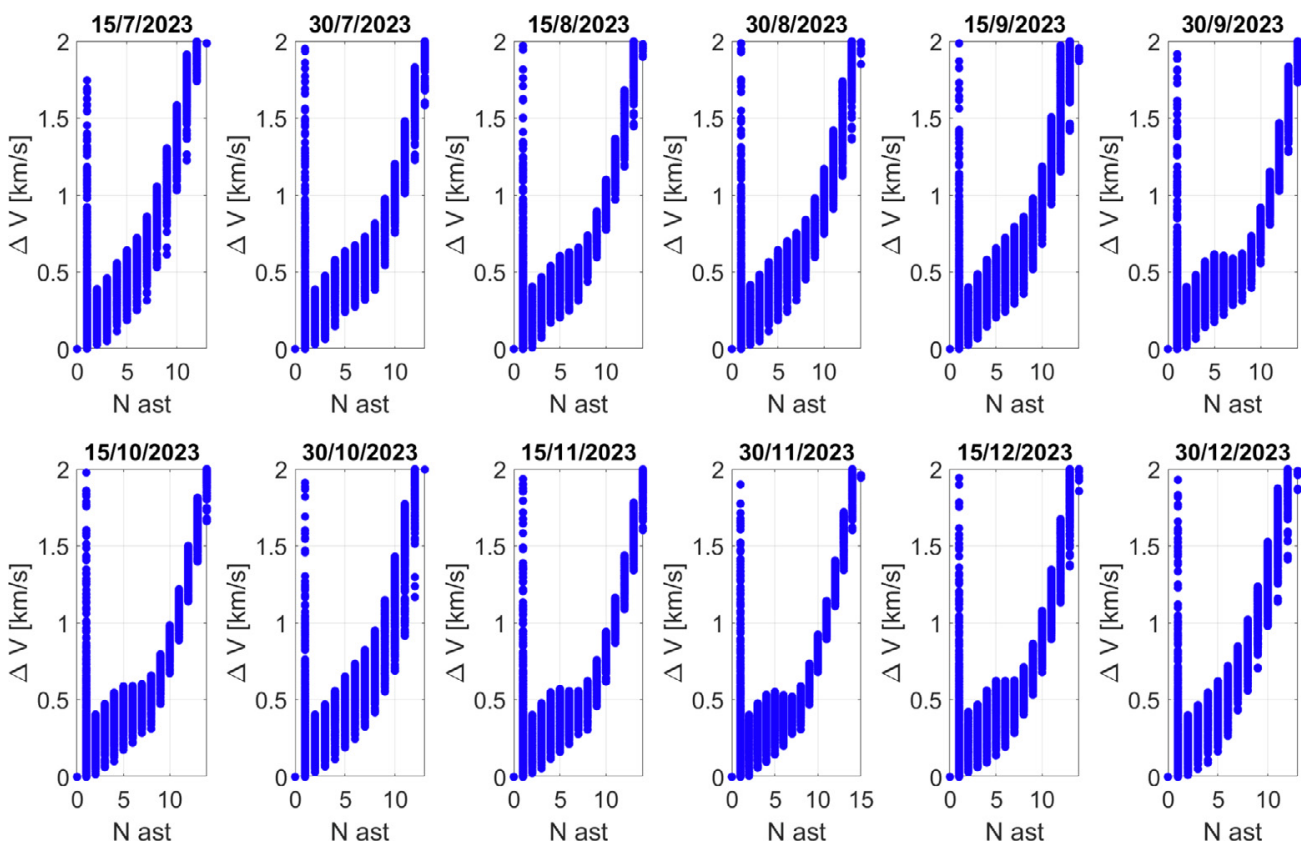

Fig. A.35. Solutions for orbit 4, from July to December 2023. 

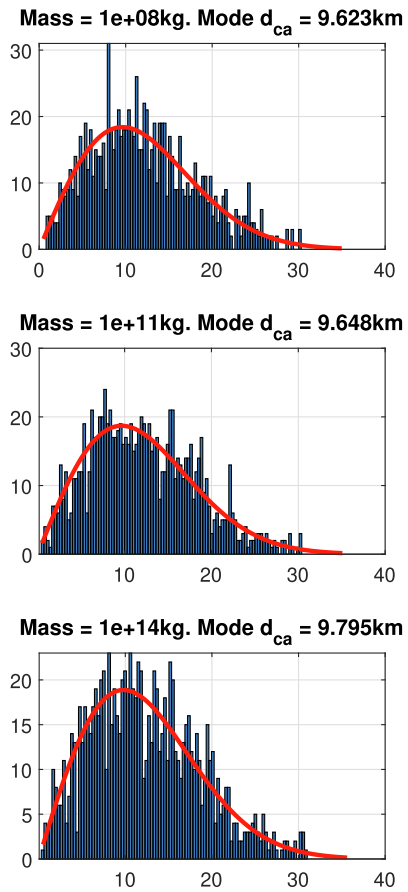
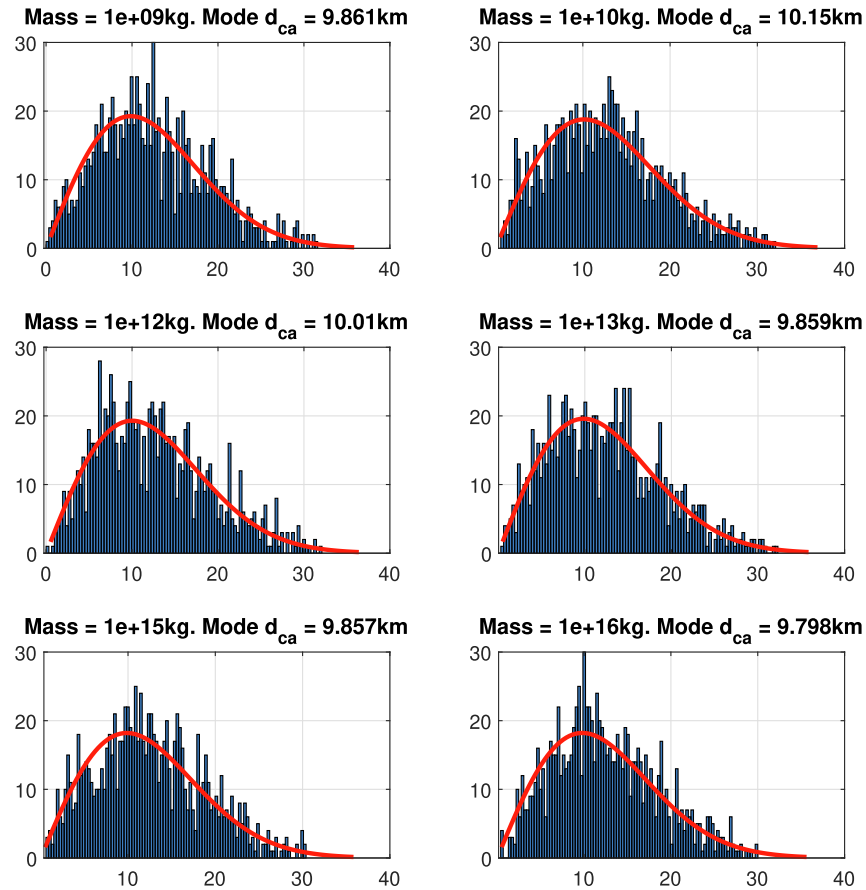

Fig. A.36. Separated histograms for the initial condition sensitivity analysis.

\section{References}

Accomazzo, Andrea, Ferri, Paolo, Lodiot, Sylvain, Hubault, Armelle, Porta, Roberto, Pellon-Bailon, Jose-Luis, 2010. The first Rosetta asteroid flyby. Acta Astronaut. 66 (3-4), 382-390.

Bessell, M.S., 1979. UBVRI photometry II: the Cousins VRI system, its temperature and absolute flux calibration, and relevance for twodimensional photometry. Publ. Astron. Soc. Pac. 91 (543), 589-607.

Bottke, William F., Vokrouhlicky, David, Rubincam, David P., Nesvorny, David, 2006. The Yarkovsky and YORP effects: implications for asteroid dynamics. Annu. Rev. Earth Planet. Sci. 34 (1), 157-191.

Center for Near Earth Object Studies, 2019. URL: https://cneos. jpl.nasa.gov/stats/ (visited on 05/01/2019).

Chesley, Steven R., Chodas, Paul W., Milani, Andrea, Valsecchi, Giovanni B., Yeomans, Donald K., 2002. Quantifying the risk posed by potential Earth impacts. Icarus 159 (2), 132-423.

Cui, Wen, Zhu, Kai-Jian, 2014. Comparison of three niters in asteroidbased autonomous navigation. Res. Astron. Astrophys. 14 (3), 329344.

Carlo, Di, Marilena, Juan Manuel, Martin, Romero, Vasile, Massimiliano, 2018. CAMELOT: computational-analytical multi-fidElity lowthrust optimisation toolbox. CEAS Space J. 10 (1), 25-36.

Carlo, Di, Marilena, Martin Romero, Juan, Manuel, Gomez, Natalia Ortiz, Vasile, Massimiliano, 2017. Optimised low-thrust mission to the Atira asteroids. Adv. Space Res. 59 (7), 1724-1739.

Carlo, Di, Marilena, Massimiliano Vasile, Dunlop, Jamie, 2018. Lowthrust tour of the main belt asteroids. Adv. Space Res. 62 (8), 20262045.

Duarte, Frank J., 2017. Tunable Laser Optics. CRC Press, Boca Raton.

Epsilon Launch Vehicle User's Manual, 2019. URL: https://global.jaxa. $\mathrm{jp} /$ projects/rockets/epsilon/pdf/EpsilonUsersManuaiy.5C e.pdf (visited on $05 / 01 / 2019$ ).

Falcon User's Guide, 2019. URL: https://www.spacex.com/sites/ spacex/files/falcon7_05C_users7o5C_guide7.5C_107.5C_2019.pdf (visited on 05/01/2019).

Funase, Ryu, Koizumi, Hiroyuki, Nakasuka, Shinichim Kawakatsu, Yasuhiro, Fukushima, Yosuke, Tomiki, Atsushim, Kobayashi, Yuta, Nakatsuka, Junichi, Mita, Makoto, Kobayashi, Daisuke, et al., 2014. 50kg-class deep space exploration technology demonstration microspacecraft PROCYON. In: 28th Annual AIAA/USU Conference on Small Satellites, pp. 4435-1435.

Greco, Cristian, Di Carlo, Marilena, Walker, Lewis, Vasile, Massimiliano, 2018. Analysis ofNEOs Reachability with Nano-Satellites and LowThrust Propulsion. In: Presentation at 4S Symposium, Sorrento, Italy.

Gronchi, Giovanni, 2005. An algebraic method to compute the critical points of the distance function between two Keplerian orbits. Celestial Mech. Dyn. Astron. 93 (1-4), 295-329.

Gronchi, Giovanni F., 2002. On the stationary points of the squared distance between two ellipses with a common focus. SIAM J. Sci. Comput. 24 (1), 61-80.

Gruen, D., Seitz, S., Bernstein, G.M., 2014. Implementation of robust image artifact removal in SWarp through clipped mean stacking. Publ. Astron. Soc. Pac. 126 (936), 158-169.

Hashimoto, Tatsuaki, Kubota, Takashi, Kawaguchi, Jun'ichiro, Uo, Masashi, Shirakawa, Kenichi, Kominato, Takashi, Morita, Hideo, 2010. Vision-based guidance, navigation, and control of Hayabusa spacecraft-Lessons learned from real operation. IFAC Proc. Vol. 43 (15), 259-264.

HNSKYPlanetarium Program, 2020. URL: https://www.hnsky.org/star_count.htm (visited on 09/25/2020).

Itzler, Mark A., Ben-Michael, R., Hsu, C.-F., Slomkowski, Krystyna, Tosi, Alberto, Cova, Sergio, Zappa, Franco, Ispasoiu, Ra.du., 2007. Single photon avalanche diodes (SPADs) for $1.5 \mathrm{ju}$ m photon counting applications. J. Mod. Opt. 54 (2-3), 283-304.

Itzler, Mark A., Jiang, Xudong, Entwistle, Mark, Slomkowski, Krystyna, Tosi, Alberto, Acerbi, Fabio, Zappa, Franco, Cova, Sergio, 2011. Advances in InGaAsP-based avalanche diode single photon detectors. J. Mod. Opt. 58 (3-4), 174-200.

JPL Small Body Database, 2019. URL: https://ssd.jpl.nasa.gov/sbdb.cgi (visited on 09/03/2019).

Leiter, Hans, Altmann, Christian, Kukies, Ralf, Porst, Jan-Patrick, 2015. Presentation at the 10th IAA Symposium on Small Satellites for Earth Observation.

Lightholder, Jack, Thompson, David R., Castillo-Rogez, Julie, Basset, Christophe, 2019. Near Earth Asteroid Scout CubeSat science data retrieval optimization using onboard data analysis. In: IEEE Aerospace Conference. IEEE, pp. 1-7. 
Luo, Hanjun, Xu, Benlian, Xu, Huigang, Chen, Jingbo, Fu, Yadan, 2015. Maximum detection range limitation of pulse laser radar with Geigermode avalanche photodiode array. J. Mod. Opt. 62 (9), 761-768.

Machuca, P., Sanchez, J.P., Masdemont, J.J., Gomez, G., 2020. Highfidelity trajectory design to flyby near-Earth asteroids using CubeSats. Acta Astronaut. 167, 146-163.

Mason, James Paul, Baumgart, Matt, Rogler, Bryan, Downs, Chloe, Williams, Margaret, Woods, Thomas N., Palo, Scott, Chamberlin, Phillip C., Solomon, Stanley, Jones, Andrew, et al., 2017. MinXSS-1 CubeSat on-orbit pointing and power performance: the first flight of the Blue Canyon technologies XACT 3-axis attitude determination and control system. J. Small Satellites 6 (12), 651-662.

McNutt, Leslie, Johnson, Les, Kahn, Pater, Castillo-Rogez, Julie, Frick, Andreas, 2014. Near-earth asteroid (NEA) scout. In: AIAA Space 2014 Conference and Exposition, pp. 1441-4435.

MPS-130 Innovative Propulsion Solutions for SmallSats, 2020. URL: https://www.rocket.com/sites/default/files/documents/CubeSat/MPS1307.5C7.20data7.5C7.20sheet7.5C7.20crop.pdf (visited on 10/05/ 2020).

NASA SSDC Archive, 2020. URL: https://nssdc.gsfc.nasa.gov/ (visited on $06 / 02 / 2020$ ).

Ozaki, Naoya, Kawabata, Yosuke, Takeuchi, Hiroshi, Ichikawa, Tsutomu, Taniguchi, Sho, Yagami, Tomoko, Funase, Ryu, Kawakatsu, Yasuhiro, 2016. Flyby navigation and guidance experiment for interplanetary micro-spacecraft PROCYON. In: 55th Annual Conference of the Society of Instrument and Control Engineers of Japan (SICE). IEEE, pp. 654-659.

PSLVSpecifications, 2019. URL: https://www.isro.gov.in/launchers/pslv (visited on 05/01/2019).

Schroeder, Daniel J, 1999. Astronomical Optics. Elsevier, Amsterdam.

Snodgrass, Colin, Jones, Geraint H, 2019. The European Space Agency's Comet Interceptor lies in wait. Nature Communications 10 (1), 1-4.

Sun, Xiaoli, Neumann, Gregory A., Abshire, James B., Zuber, Maria T., 2006. Mars $1064 \mathrm{~nm}$ spectral radiance measurements determined from the receiver noise response of the Mars Orbiter Laser Altimeter. Appl. Opt. 45 (17), 3960-3971.

Tardioli, C., Farnocchia, D., Rozitis, B., Cotto-Figueroa, D., Chesley, S. R., Statler, T.S., Vasile, M., 2017. Constraints on the near-Earth asteroid obliquity distribution from the Yarkovsky effect. Astron. Astrophys. 608 (A61), 1-9.

Tedesco, Edward F., 1994. IRAS minor planet survey. In: International Astronomical Union Symposium, vol. 160. Cambridge University Press, pp. 463-166.

Thiry, Nicolas, Vasile, Massimiliano, 2017. Statistical multi-criteria evaluation of non-nuclear asteroid deflection methods. Acta Astronaut. 140, 293-307.

Vetrisano, Massimo, Vasile, Massimiliano, 2016. Autonomous navigation of a spacecraft formation in the proximity of an asteroid. Adv. Space Res. 57 (8), 1783-1804.

Virgin Orbit Service Guide, 2019. URL: https://virginorbit.com/wpcontent/uploads/2018/11/Virgin-Orbit-Service-Guide.pdf (visited on 05/01/2019).

Walker, Lewis, Greco, Cristian, Di Carlo, Marilena, Wilson, Andrew, Ricciardi, Lorenzo, Berquand, Audrey, Vasile, Massimiliano, 2019. Nanospacecraft exploration of asteroids by collision and flyby reconnaissance. In: Low-Cost Planetary Missions Conference.

Walker, Roger, Detlef, Koschny, Chiara, Bramanti, 2017. Miniaturised Asteroid Remote Geophysical Observer (M-ARGO): a stand-alone deep space CubeSat system for low-cost science and exploration missions. In: Presentation at 6th Interplanetary CubeSat Workshop, Cambridge, UK.

White, Richard L., Helfand, David J., Becker, Robert H., Glikman, Eilat, de Vries, Wim, 2007. Signals from the noise: image stacking for quasars in the FIRST survey. Astrophys. J. 654 (1), 99-114.

Wolters, Stephen D., Ball, Andrew J., Wells, Nigel, Saunders, Christopher, McBride, Neil, 2011. Measurement requirements for a NearEarth Asteroid impact mitigation demonstration mission. Planet. Space Sci. 59 (13), 1506-1515. 